\title{
Review Article \\ Singlet Generation from Triplet Excitons in Fluorescent Organic Light-Emitting Diodes
}

\author{
A. P. Monkman \\ Organic Electroactive Materials Research Group, Department of Physics, University of Durham, Durham DH1 3LE, UK \\ Correspondence should be addressed to A. P. Monkman; a.p.monkman@durham.ac.uk
}

Received 22 August 2012; Accepted 4 October 2012

Academic Editors: I. A. Hümmelgen and E. J. Nassar

Copyright ( 2013 A. P. Monkman. This is an open access article distributed under the Creative Commons Attribution License, which permits unrestricted use, distribution, and reproduction in any medium, provided the original work is properly cited.

\begin{abstract}
A potential major drawback with organic light-emitting devices, (OLEDs) is the limit of 25\% singlet exciton production through spin-dependent charge recombination. Recent device results, however, show that this limit does not hold and far higher efficiencies can be achieved in purely fluorescent-based systems (Wohlgenannt et al. (2001), Dhoot et al. (2002), Lin et al. (2003), Wilson et al. (2001), Cao et al. (1999), Baldo et al. (1999), and Kim et al. (2000)). Thus, the question arises; is recombination spin dependent (Tandon et al. (2003)) or are singlet excitons generated in secondary processes? Direct measurement of the singlet generation rate in working devices of $44 \%$ has been shown (Rothe et al. (2006)), which have been verified as being part due to direct singlets formed on recombination and part from triplet fusion, singlets produced during triplet annihilation (Kondakov et al. (2009), King et al. (2011), and Zhang and Forrest (2012)). Here, the various routes by which triplet excitons can generate singlet states are discussed and their relative contributions to the overall electroluminescence yield are given. The materials requirements to obtain maximum singlet production from triplet states are discussed. These triplet contributions can give very high device yields for fluorescent emitters, which in the case of blue devices can be highly advantageous. Further, new devices architectures open up which are simple and have intrinsically low turn on voltages, ideal for large-area OLED lighting applications.
\end{abstract}

\section{Introduction}

Current state-of-the-art OLED and PLED devices have been optimised for use in displays, having small-area pixels, yielding high efficiency and good individual colour from each pixel. Active matrix displays using these are now found in the latest smart phones (ca. 2012), including Samsung's fastest selling smart phone to date, the Galaxy S2. The displays employ red phosphorescent emitters but blue and green fluorescent emitters. In the latest generation of high-efficiency ( $>60 \mathrm{~lm} / \mathrm{W}$ ) organic solid-state lighting (OSSL) panels from Novaled, Osram, and Konica Minolta-Philips, use of all phosphorescent emitters yields very warm white colours, with poor colour temperatures of $2600 \mathrm{~K}$, and poor lifetimes especially for the blue component [1-3]. The phosphorescent emitters lack good saturated colour but more importantly the blue metal organic complexes used are unstable. For the most common blue (aqua) phosphor, FIrpic [4], vacuum deposition causes partial loss of fluorine substituents from the ligands, and partial decomplexation of the picolinate (pic) ancillary ligand $[5,6]$, this then causes further degradation over a short period of running, leaving the blue pixels with a much shorter lifetime than both the red and green. Secondly, because of the necessity of having such a high triplet energy to yield the correct blue colour, problems arise with finding a host material with a triplet energy higher than that of the emitter but which can also act as a good transport material for electrons and holes [7-11]. Currently, the best state-ofthe-art blue phosphorescent material is that demonstrated by BASF and the group of Kido at Yamagata University [12], who report a "blue" phosphorescent device from a nonfluorine containing Ir complex having peak emission at $454 \mathrm{~nm}$ with high quantum efficiency $36 \mathrm{~lm} / \mathrm{W}, 29 \mathrm{~cd} / \mathrm{A}$, EQE 18.6\%, but which suffers from serious efficiency roll off. As yet, only poor lifetimes have been achieved, which suggest that it too has degradation problems, probably arising from the triplet energy being close to that required to break the ligand bonds, that is, a fundamental limitation. For PLED systems, the problem with blue phosphors is the fact that only few layer devices can be fabricated by sequentially solution deposition 
[13-18] so the high triplet energy host materials required cause serious charge injection problems. Although many groups are currently trying to tackle this problem, developing new materials sets for blue phosphorescent emitters using simple few layer device structures, alternatives to blue phosphorescent OLEDs are being sort. There is still great potential to carry on using fluorescent blue emitters, for both display and white light applications where lifetime is the most demanding and critical parameter for the devices, but we would still require a way to make use of (75\%) triplet excitons formed in the fluorescent emitters. Several ideas have emerged on triplet harvesting from a blue fluorescent emitter into red and green phosphors [19-21]; however, the device structures required to achieve this are very complex and involve exciton blocking layers of order $2 \mathrm{~nm}$ thick, totally impractical to manufacture at high yield over the large areas required for lighting. These complex device structures are not used in the current generation lighting panels or displays. Thus, a new triplet harvesting approach is required. It is well known that triplet excitons can be used to derive useful single excitons from triplet-triplet annihilation (TTA) by the process of triplet fusion (TF) [22-26]. When two triplet excitons interact, and depending on the subsequent spin configuration of the resultant "interaction pair," they can produce a singlet exciton (TF), further triplet excitons or quintet states [27]. Historically, a pure spin statistically argument has been used to imply that only 1 singlet exciton is produced from 18 initially interacting triplets [27-29], but in most molecules the quintet states are energetically untenable [30-32] and in a few specific molecules the upper excited triplet state, $T_{n}$, cannot be reached either, as with rubrene [33, $34]$, given the sum of the energy of the two triplet excitons. Thus, the number of singlet excitons produced via TF can be much higher than $1 / 18$, allowing fluorescent dopants to be effectively used as triplet "harvesting" emitters, yielding longer device lifetimes and removing the stringent requirements of high triplet energy host materials required with blue phosphorescent dopants. In this paper, the processes and various different ways in which singlet excitons can be generated from triplet states are explained.

The factors which control the overall quantum efficiency of an organic light-emitting diode are rather different than those that govern inorganic LEDs efficiency, most notably the ratio of singlet to triplet excitons formed as a result of charge recombination within the organic emitter layer. In the organic semiconductors, the singlet and triplet excitons are very well resolved with little intermixing [35]. This difference comes about because of the excitonic nature of the fundamental excited states of the organic emitters and the large electron correlation energies of the molecules [36, 37]. Since the electron and hole are tightly bound with large exciton binding energy [38-40], singlet excitons are spatially localized and so their exchange energy is large, whereas for the triplet excitons the electron and hole are in orthogonal orbital's so the triplet excitons have a zero exchange term [41, 42]. This manifests itself in the very large difference in the energies of the lowest singlet $\left(S_{1}\right)$ and triplet $\left(T_{1}\right)$ excited states (excitons), experimentally found to be $T_{1}=\left(1.13 \times S_{1}-1.43\right) \pm 0.15 \mathrm{eV}$ (typically of order $0.7 \mathrm{eV}$ ) and conjugation length dependent $[37,43]$.
The singlet excitons are also strongly coupled to the ground state giving rise to high photoluminescence quantum yields. Further, as organic semiconductors typically contain only low atomic mass elements, spin orbit coupling, the dominant mechanism for triplet formation $[35,44]$ is weak, and so the interconversion of singlet excitons into triplet excitons, that is, intersystem crossing, is very inefficient $[45,46]$, again helping to achieve high photoluminescence quantum yields. Thus, to understand the charge recombination process in OLEDs we must also take into account these excitonic properties.

\section{Exciton Formation in an OLED}

Charges of opposite sign injected into the organic semiconductor form dressed states not free electrons and holes, the charge perturbs the covalent $\pi$ bonding structure of the organic semiconductor creating a localized distortion which traps the charge, that is, a polaron (P) [47], and it is these polarons (positive and negative) that migrate through the organic layer to meet in a thin recombination zone $[48,49]$. These polarons are spin $1 / 2$ particles. When oppositely charge polarons capture, they initially form some intermediate state, held by their mutual columbic potential well [50], before relaxing into an exciton (which can then emit). Before capture, the polarons are uncorrelated and so their spin states have random orientation with respect to one another. Only at the point of recombination do the two spins of the $\mathrm{P}^{+}$and $\mathrm{P}^{-}$become correlated and singlet or triplet character can be associated to the intermediate state [51]. The intermediate state can thus be best described as either a singlet or triplet charge transfer (CT) state (or charge transfer exciton) [52]. If this recombination process is, thus, independent of the spin states $(\downarrow)$ of the initial $\left(\mathrm{P}^{+} \mathrm{P}^{-}\right)$pair, then quantum mechanical spin statistics dictates that there are four ways in which the spin wavefunctions of the individual polarons can combine when the CT excitonic state is formed, that is, $\uparrow+\uparrow$ (triplet), $\downarrow+\downarrow$ (triplet), $1 / \sqrt{2}[\uparrow \downarrow+\uparrow \downarrow]$ spins precessing in-phase (triplet), $1 / \sqrt{2}[\uparrow \downarrow$ - $\uparrow \downarrow]$ spins precessing out of phase (singlet). From this, it can be seen that only $25 \%$ of the excitons thus created will have singlet character and be emissive which puts an upper limit of 0.25 on the internal quantum efficiency of an OLED. However, this limit only arises if the recombination process is independent of spin. If at any stage the recombination is effected directly or indirectly by the spin configuration of the intermediate CT states [53] or the polaron capture is affected by their spins [54], then it can follow that the $25 \%$ limit is broken and possibly more singlets could be produced [21].

Over the past few years, an increasing number of experimental reports make it clear that the $25 \%$ limit is broken [5559]. In small-molecule-based OLEDs, original experimental evidence pointed to the $25 \%$ limit being obeyed $[60,61]$, whereas in polymers this was not so; however, more recent reports, especially those from the OLED group in Kodak have shown that the $25 \%$ limit in small-molecule devices was also greatly exceeded $[62,63]$. Many theories were put forward as 
to why there might be such a difference in the fundamental physics of these two different materials based systems.

From the first experimental results showing greater than 0.25 singlet yield in polymers, models of the charge recombination process, which predicted spin-dependent recombination, were developed. The earliest model was put forward by Tandon et al. [64], modelling the initial polymer based results of Wohlgenannt et al. $[55,65]$. Their model assumes that the recombination process initially occurs on two neighbouring chains, forming an interchain CT exciton [66] before a final exciton forms on one chain. They show that in a conjugated polymer both the ground state and the lowest excited triplet state (exciton wavefunction) are covalent, whereas the singlet excited state wavefunction is made from a combination of covalent and charge separated (ionic) configurations, that is, doubly occupied sites as originally put forward in Simpson's "valance bond" model $[67,68]$. Thus, when a $\mathrm{P}^{+}$and $\mathrm{P}^{-}$ (on neighbouring chains) initially capture and form an intermediate "charge transfer" (CT) state, this too must have ionic character; so there should be a higher probability to form the singlet exciton than the covalent triplet exciton from the intermediate CT state. This stems from Fermi's Golden rule, the bigger the overlap of the initial and final state wavefunction the greater the rate of the transition. Thus because of the large singlet electron exchange energy, the ionic singlet wavefunction is more delocalized, that is, the two electrons cannot occupy the same site, than the localized triplet, and so the diffuse (ionic) intermediate CT state will more readily decay into the singlet channel than the triplet. This idea then readily explains why in the smallmolecule systems the spin statistical limit is preserved as here there is very little difference between the spatial extent of the singlet and triplet wavefunctions. However, recent indepth analysis of CT states in luminescent polymers [54, $69,70]$ shows that the decay channels for CT states follow the inverse of this behaviour, which is outlined later in the paper.

At the same time as this, Karabunarliev and Bittner $[52,71]$ proposed a different model, again assuming an intermediate CT state but they considered the whole recombination process to occur on a single chain. In this model, the polarons capture on a single chain, the wavefunction of the CT state initially formed is a $1: 1$ superposition of covalent and ionic configurations yielding 1:1 singlet and triplet CT states. Because the energies of both the CT states are roughly equal (true in certain cases), the relaxation from a singlet CT state to a singlet exciton state goes at a faster rate because less energy (phonons) need to be given off, noting the exchange interaction yields triplet excitons $0.7-1 \mathrm{eV}$ [37] lower in energy than singlet excitons. Thus, singlets are formed at a faster rate than triplets. From their calculations, the ratio of formation rates (singlet to triplet) is chain-length-dependent and for short chains the rates of exciton formation become equal, as the singlet triplet energy gap equalize $[42,43]$, yielding a $25 \%$ singlet exciton generation fraction, as seen in small molecules. Thus, given an initial 1:1 singlet to triplet CT formation rate on polaron recombination, the relaxation to excitons is controlled by the number of phonons emitted in relaxing down to the respective exciton and in polymers because the triplet state is at least $0.7 \mathrm{eV}$ below that of the singlet, the singlets are made faster. This theory faces two major problems, however, firstly, in small molecules the triplet exciton is again between 0.5 and $0.8 \mathrm{eV}$ below that of the singlet exciton [72, 73] hence the model cannot predict the correct small molecule behaviour and second it again does not take into account the fact that the triplet state of the polymer must be below that of the CT triplet state which gives a very rapid quenching channel for the CT states to the triplet exciton of the polymer, especially when the CT singlet and triplet states are close in energy as required by the theory, and noting the CT singlet must be energetic enough to create a polymer singlet state.

Many others have followed on from Karabunarliev and Bittner's work, using their model as a starting point. Yin et al. [74] have suggested that an applied electric field, such as we have in a working device would lower the energy of the CT states. This would bring the singlet CT state closer into resonance with the singlet exciton and thus enhance the singlet channel over the triplet channel. Barford [50] came to a very similar conclusion but also showed that because the Frank-Condon overlap between the phonon modes in the triplet manifold are smaller than for the singlets, the relaxation rate of the triplet $\mathrm{CT}$ into the exciton is slowed even more. Das et al. [75], Beljonne et al. [76, 77], and Chen et al. [78] come to similar conclusion as that of Karabunarliev and Bittner.

As already mentioned, recent studies on CT states in luminescent polymers and small molecules casts severe doubts on these models based on an intermediate CT state. Firstly, as with all $\mathrm{CT}$ systems the $\mathrm{CT}^{3}$ rapidly decays to a lower lying triplet exciton of the donor-acceptor pair (forming the CT state) [79] whereas $\mathrm{CT}^{1}$ has much more complicated decay channels. $\mathrm{CT}^{1}$ can cross to $\mathrm{CT}^{3}$ with typically an enhanced ISC (compared to the donor) [46]. Those $\mathrm{CT}^{1}$ which decay to the $S^{1}$ can also suffer quenching to the $T^{1}$ state via normal ISC. The key parameters dictating $S^{1}$ formation are, thus, the competition between the $\mathrm{CT}^{1} \rightarrow$ $\mathrm{CT}^{3}$ interconversion rate, the $\mathrm{CT}^{1} \rightarrow S^{1}$ formation rate, and $S^{1} \rightarrow T_{1}$ ISC rate. We know that for most emissive polymers ISC is rather slow, of order $10^{6} \mathrm{~s}^{-1}$ [80]; so radiative decay will dominate but ISC within the CT state is higher than this and so some $\mathrm{CT}^{1}$ will cross to $\mathrm{CT}^{3}$. Thus, $25 \%$ singlet yield must be considered the theoretical maximum and in practice a lower singlet yield would be found. Further more, in most materials the CT states are lower in energy than the $\pi$ states so can not form "singlet states".

\section{Experimental Observations}

The majority of early $S: T$ ratio measurements on polymeric semiconductors were based on photophysical measurements on the emissive materials, not devices, and have given ratios ranging from 33 to $62 \%$ depending on the polymer backbone structure $[55,56,65]$. The validity of assumptions made or estimated absolute values of many quantities that 
are difficult to obtain are questionable, for example, the basis of the magnetic resonance measurements used by Wohlgenannt et al. [55] which initially attracted considerable attention, have recently been seriously challenged [ 81 , 82]. The nature of excited state species and interactions that optically detected magnetic resonance (ODMR) and photoluminescence detected magnetic resonance (PLDMR) actually detect is not clear. For example, Segal et al. [82, 83] have proposed the importance of PL quenching arising from singlet (and triplet) exciton polaron interactions in these polymers, a process that we have shown to be efficient in working PLED devices [84, 85]. Further studies have tried to determine the relative singlet to triplet yield by employing emissive (phosphorescent) acceptors $[60,86]$. These, however, are not measurements on the pristine polymer and uncertainties remain as to what extent the intersystem-crossing yield, $\kappa^{\mathrm{ISC}}$, in such doped systems is still a meaningful constant [87] as it has been shown that the heavy atom dopants seriously perturb the spin orbit coupling of the polymer backbone, greatly enhancing the polymer ISC rate. Furthermore, the implied equivalence of optically and electrically excited excitons in these experiments may not hold true [88], given the fact that common phosphorescent acceptors are electron traps and thus act as recombination centres only during electrical excitation [89]. Apart from such indirect materials approaches, experiments that directly probe the number of singlet and triplet excitons formed from charge carrier recombination are generally more convincing and transparent [90]. Attempts to determine absolute values are certainly unrealistic $[56,91,92]$, given the experimental difficulties in collecting all the emission from a device, especially waveguided light, and the difficulty in measuring the true current which yields light generation rather than IR losses in the ITO and capacitance effects, are unknown. Instead, relative measurements are more appropriate methods and the general approach we have taken is outlined as follows.

Generally, we can define two parameters, $S$ and $T$, which represent the experimentally measurable signals that are proportional to the singlet and triplet generation rates and each $c_{i}$ denotes appropriate constant of proportionality to account for the collected signal, that is, representing the experimental collection cone. The superscripts el and opt distinguish electrical and optical excitation. Given that a certain electrical (optical) excitation causes exciton formation, $I$, then the notionally observable signals are given as

$$
\begin{gathered}
S^{\mathrm{opt}}=c_{S}^{\mathrm{opt}} I_{S}^{\mathrm{opt}}\left(1-\kappa^{\mathrm{ISC}}\right), \\
T^{\mathrm{opt}}=c_{T}^{\mathrm{opt}} I_{T}^{\mathrm{opt}} \kappa^{\mathrm{ISC}}, \\
S^{\mathrm{el}}=c_{S}^{\mathrm{el}} I_{S}^{\mathrm{el}} \chi\left(1-\kappa^{\mathrm{ISC}}\right), \\
T^{\mathrm{el}}=c_{T}^{\mathrm{el}} I_{T}^{\mathrm{el}}\left(1-\chi\left(1-\kappa^{\mathrm{ISC}}\right)\right),
\end{gathered}
$$

with $\chi$ being the singlet generation yield. By using the same excitation conditions for the singlet and triplet measurements, that is, $I_{S}^{\mathrm{opt}}=I_{T}^{\mathrm{opt}}$ and $I_{S}^{\mathrm{el}}=I_{T}^{\mathrm{el}}$, one can measure $T$ relative to $S$ :

$$
\begin{gathered}
\frac{T^{\mathrm{opt}}}{S^{\mathrm{opt}}}=\frac{c_{T}^{\mathrm{opt}}}{c_{S}^{\mathrm{opt}}} \frac{\kappa^{\mathrm{ISC}}}{\left(1-\kappa^{\mathrm{ISC}}\right)}, \\
\frac{T^{\mathrm{el}}}{S^{\mathrm{el}}}=\frac{c_{T}^{\mathrm{el}}}{c_{S}^{\mathrm{el}}} \frac{1-\chi\left(1-\kappa^{\mathrm{ISC}}\right)}{\chi\left(1-\kappa^{\mathrm{ISC}}\right)} .
\end{gathered}
$$

In this case, neither the driving current, including dark currents, nor the optical excitation dose (intensity actually absorbed by the semiconductor) need to be known. Furthermore, if $S^{\mathrm{opt}}$ (or $T^{\mathrm{opt}}$, resp.) is probed under the same experimental conditions (geometry) as $S^{\mathrm{el}}$ (or $T^{\mathrm{el}}$ ), then $c_{S}^{\mathrm{opt}}=c_{S}^{\mathrm{el}}\left(c_{T}^{\mathrm{opt}}=c_{T}^{\mathrm{el}}\right)$ and one measures the electrically excited signals relative to the corresponding optical ones:

$$
\frac{T^{\mathrm{el}} / S^{\mathrm{el}}}{T^{\mathrm{opt}} / S^{\mathrm{opt}}}=\frac{1-\chi\left(1-\kappa^{\mathrm{ISC}}\right)}{\chi^{\mathrm{ISC}}} .
$$

Here the absolute value of only one parameter, the intersystem-crossing yield, $\kappa^{\mathrm{ISC}}$, is required in order to calculate the singlet formation yield, $\chi$. Equation (3) is true for any optical excitation combined with any electrical one as long as (i) all signals depend linearly on excitation, (ii) the corresponding $T$ and $S$ signals are excited the same way, and (iii) the corresponding el and opt signals are measured the same way. The latter two points are satisfied using a single experimental optical layout, as shown schematically for our experiment in Figure 1. This also removes complications with collection of emitted light, for example, the light outcoupling efficiency is the same for all measurements [93]. Also this condition accounts for all exciton quenching mechanism as long as they apply in the same way for optically and electrically excited excitons. For example, quenching at the anode or impurity sites including the well-known (photooxidative) keto defect $[94,95]$. It is known that keto defects act as charge traps for electrical excitation, but this is still not a problem since it reduces the singlet and triplet density proportionally similar to a dark current.

By way of example, we have studied polyspirobifluorene in detail, this was synthesized by Merck OLED GmbH [96]. Spirofluorene derivatives are chemically inert against backbone oxidation, which otherwise causes the formation of keto defects $[94,95]$. State-of-the-art diodes were fabricated at Philips Laboratories, Eindhoven using ITO and $\mathrm{Ba} / \mathrm{Al}$ as electrode materials. A hermetically sealed metal cap was applied as well in order to protect the cathode from oxidation, details are given in [97]. All measurements were performed at $20 \mathrm{~K}$ using an optically and electrically accessible closed cycle helium cryostat. The triplet population was determined using triplet transient absorption as a function of the time during a one millisecond excitation pulse. Without any alternations in the geometry of the spectrometer, the latter could either be electrically applied using a $100 \mathrm{~W}$ current pulse generator or optically using a $405 \mathrm{~nm}$ laser diode. A $780 \mathrm{~nm}$ probe beam 


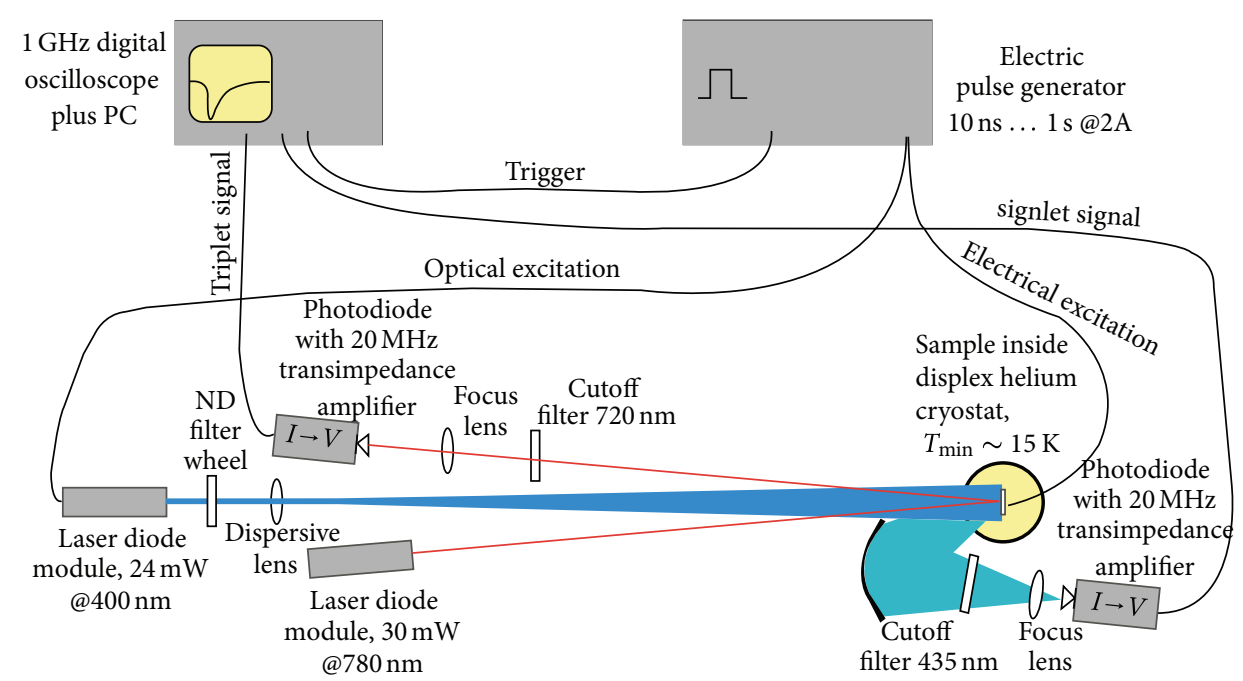

FIGURE 1: Diagrammatic optical layout of the experiment used to measure the singlet generation yield from an OLED. Simultaneous measurement of fluorescence, electroluminescence, and triplet-induced absorption, both through optical and electrical excitation of the device are made whilst operating at $20 \mathrm{~K}$ or below.

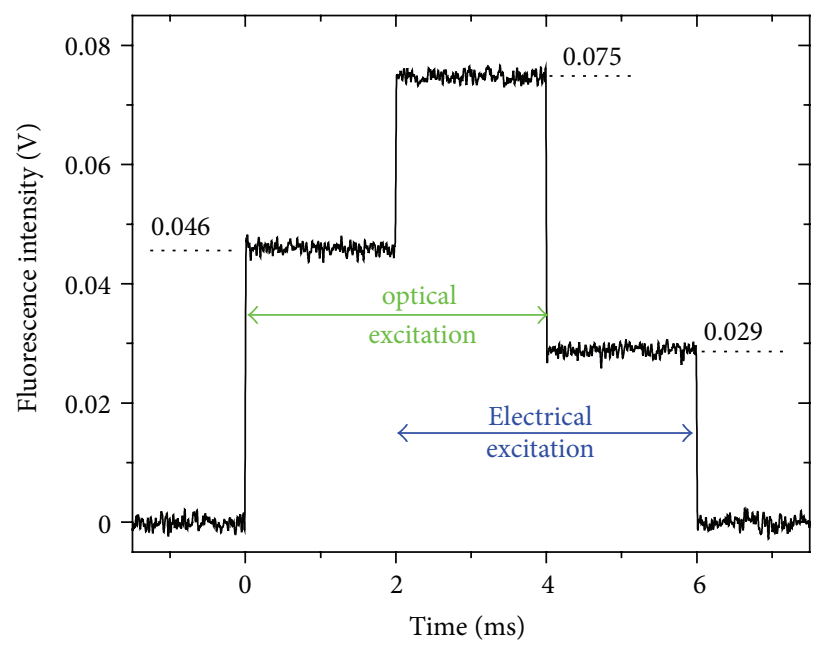

Figure 2: Demonstration of the additive nature of the simultaneous signals and lack of excitation-dependent quenching mechanisms for these measurements. Here the optical electrical and combined excitations of fluorescence from a polyspirobifluorene device are compared.

was focused onto the active area of the device, reflected by the cathode, passed through an appropriate cutoff filter, detected by a $200 \mathrm{MHz}$ transimpedance amplifier and monitored by a $1 \mathrm{GHz}$ Oscilloscope. Up to 1000 excitations were averaged for a single dataset with a repetition frequency of only $0.3 \mathrm{~Hz}$ in order to allow for sufficient triplet decay between the excitation pulses. The fluorescence level was simultaneously observed using a second photodiode.

Equation (3) holds for any pair of signals, $T$ and $S$, as long as both depend linearly on excitation dose. Fluorescence and electroluminescence intensities obviously satisfy these requirements. For both kinds of excitation (optical or electrical), these signals are truly time-independent and directly proportional to the singlet generation rates at normal excitation densities, only at high laser fluences do nonlinear effects start to emerge [98]. This can be seen in Figure 2, where the individual optically and electrically excited signals perfectly add up to the simultaneously excited one, that is, the optically excited fluorescence contribution in the presence of the electrical excitation is unchanged. This also shows that for singlet excitons, quenching by the electric field [99] or by polarons [100] is negligible under the conditions used in our experiments. For the triplet signal, either triplet transient absorption [90,101] or phosphorescence [85] could be used. However, both require large excitation densities to yield appropriate signal-to-noise ratios, which also causes migration activated TTA a major quenching channel [102, 103]. The situation becomes (relatively) worse at higher temperature, because triplet mobility increases-at room temperature triplet excitons decay essentially only by TTA, without any phosphorescence emission $[49,104]$. Given that both emission and transient absorption detection are not 


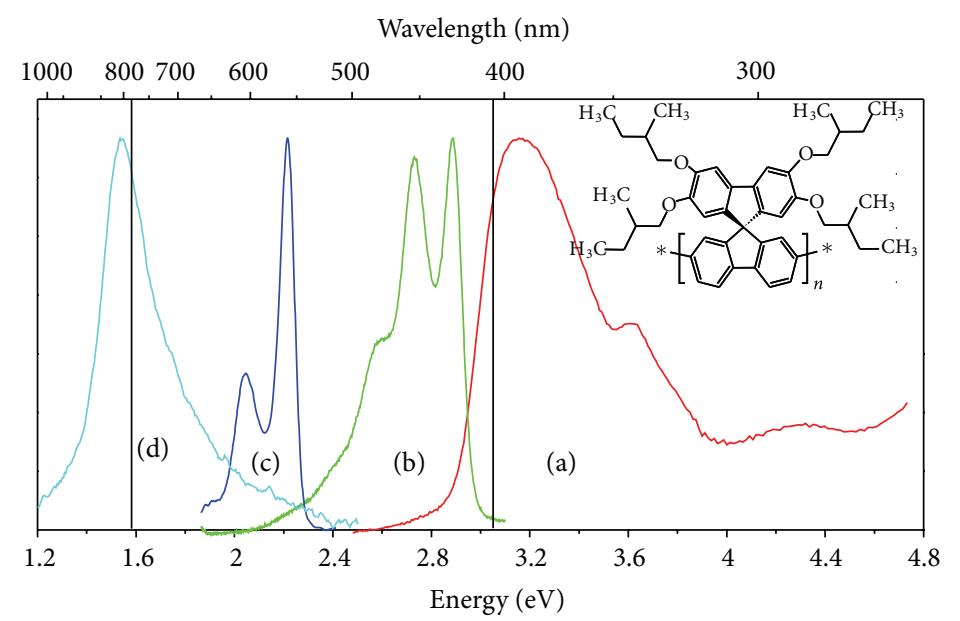

FIGURE 3: Spectroscopic properties of polyspirobifluorene indicating the optical features used in the measurement of the singlet generation ratios. Absorption (a), electroluminescence (b), electrophosphorescence (c), and transient triplet absorption spectra (d). The vertical lines indicate the energies of the optical excitation and the transient absorption probe, respectively, and the inset shows the repeat unit of the polyspirobifluorene.

ideal measurements for the triplet population, we still have to rely on them because there are no convincing alternatives.

Basic optical properties of the blue-emitting polyspirobifluorene polymer, see inset of Figure 3 for chemical structure [96], are shown in Figure 3. All experiments were performed at $20 \mathrm{~K}$, as this reduces the triplet migration to quenching sites but does prevent TTA [103]. The time-dependent triplet population density was monitored by its transient absorption [101] and Figure 4 shows two typical datasets for optical and electrical excitation. Here, we observe the build-in of the triplet excitons as a function of time during a one millisecond electrical excitation pulse. TTA gives rise to both the saturation of the induced absorption signal and the rapid decay of the triplet density in the absence of excitation compared to the long radiative lifetime of the triplet exciton, $\sim 1$ s $[102,103]$. Therefore, the observed data correspond to the accumulated (integrated) triplet density, limited only by TTA. Fitting these curves then yields the triplet generation rate and populations. The starting point for this is the rate equation for the triplet accumulation. As the triplet exciton lifetime $\sim 1 \mathrm{~s}$, within the time frame of the measurements (1 ms) we can ignore monomolecular decay (phosphorescence). If under certain continuous excitation conditions, the triplet generation rate is $I_{T}$ then the measured signal intensity given by:

$$
\begin{aligned}
& \frac{d T(t)}{d t}=\frac{1}{c_{T}}\left[I_{T}-\gamma_{T T} c_{T}^{2}(T(t))^{2}\right], \\
& T(t)=\frac{1}{c_{T}} \sqrt{\frac{I_{T}}{\gamma_{T T}}} \tanh \left(t \sqrt{I_{T} \gamma_{T T}}\right),
\end{aligned}
$$

with $\gamma_{T T}$ being the TTA constant.

A further important consideration that also has to be taken into account, both for electrical and optical excitations, is the fact that the excitons are not homogeneously formed throughout the organic semiconductor. This is obvious for optical excitation, but also for electrical excitation the dissimilar mobility of the charge carriers creates a recombination zone near to the electrode that injects the slower moving charges [105]. Given that the triplet excitons are nearly immobile at $10 \mathrm{~K}$ [103], they cannot rapidly compensate for this inhomogeneity by migration, and so the triplet population density is far higher within this narrow recombination zone. This has an immediate effect on TTA as the TTA rate depends on the triplet exciton density, and so forming them within a very thin recombination zone in the active material will give much higher TTA rates than one would expect for the same triplet population distributed throughout the whole of the organic semiconductor. The narrow recombination zone persists even at room temperature and so this picture holds at elevated temperatures as well. Modelling of such simple polymer devices shows that both the recombination zone and the optical absorption occur in similar positions close to the anode. This means that the optical cavity effects on emission from the device structure are very similar for both types of excitation mechanism. Therefore, we account for this inhomogeneous excitation profile using a simple exponential excitation profiles with characteristic inverse thickness of recombination zone, $\lambda^{\mathrm{OPT}}$ and $\lambda^{\mathrm{el}}$, respectively,

$$
\begin{gathered}
\frac{d T(x, t)}{d t}=\frac{1}{c_{T}}\left[I_{T} \lambda e^{-\lambda x}-\gamma_{T T} c_{T}^{2}(T(x, t))^{2}\right], \\
T(t)=\frac{1}{c_{T}} \frac{2}{t a} \ln \cosh \left(t \sqrt{I_{T} a}\right) \quad \text { with } a=\gamma_{T T} \lambda .
\end{gathered}
$$

This model only relies on two free fitting parameters, $1 / c_{T} a$ and $I_{T} a$, with $I_{T} / c_{T}=d T / d t$ for $t=0$ being the desired quantity. Indeed, accounting for the inhomogeneous exciton generation results in a much improved fit of the experimental data, which can only really be appreciated in the semilogarithmical presentation of the inset of Figure 4 . The slope obtained according to (5) is shown as a dashed line. We have also used this to give a more detailed analysis the charge 


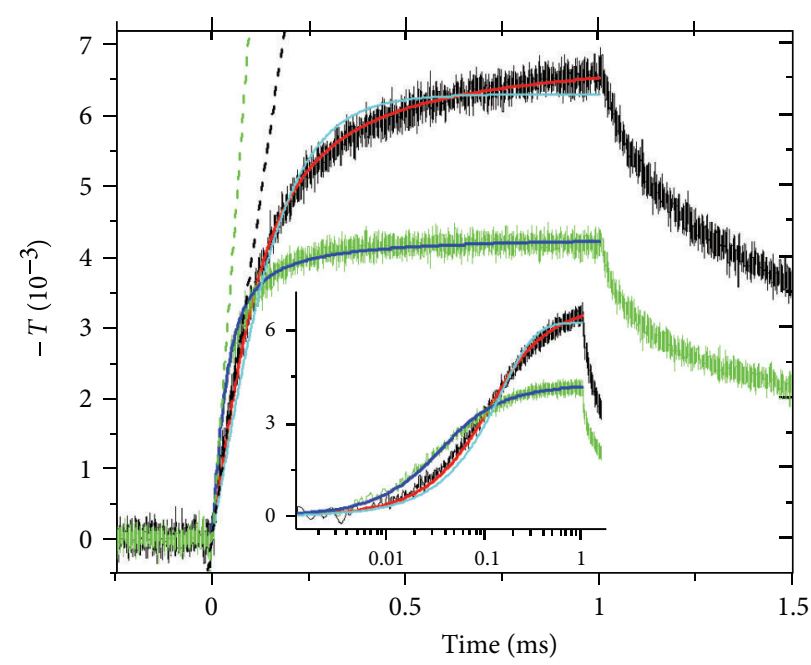

FIGURE 4: Transient triplet absorption data set measured during and after a $1 \mathrm{~ms}$ optical (black) and electrical (green) electrical pulse as a function of time. The red and blue solid lines are least square fits according to (5); the cyan line according to (4). The dashed lines indicate the slopes obtained for this particular datasets. In the inset, the same data are replotted with a logarithmic time scale.

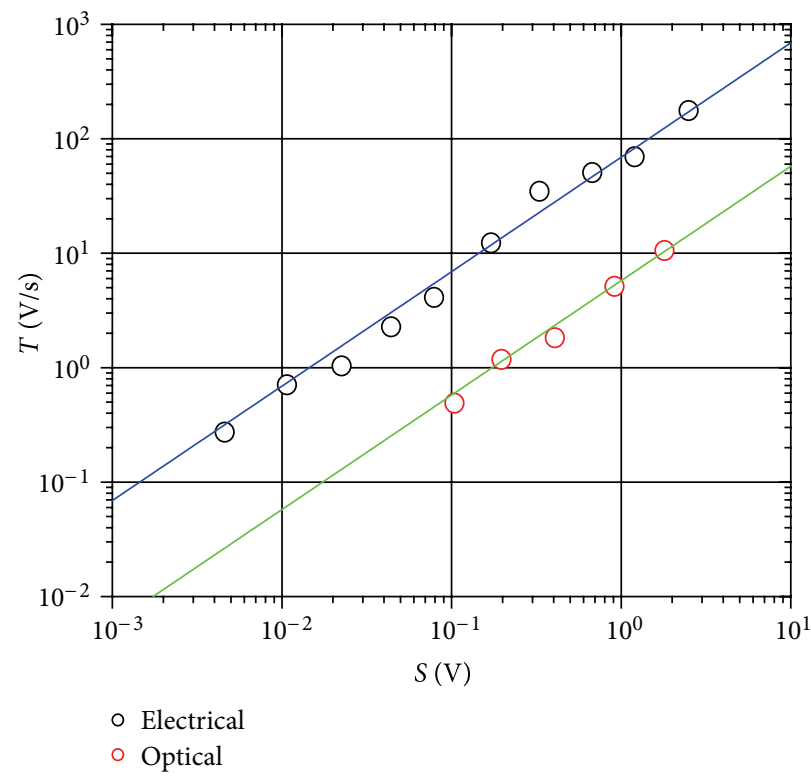

FIGURE 5: Dependency of the rise of the transient triplet absorption signal $(T)$ on its corresponding fluorescence level $(S)$ for electrical and optical excitation on a double logarithmical scale. The two solid lines are least square fits of the offset of a strictly linear dependency. Data are given in volts from the original measurement from the oscilloscope.

carrier recombination layer, $\lambda^{\mathrm{el}}$, in working devices, showing that the recombination zone is only some $5-10 \mathrm{~nm}$ thick [101]. Slopes were then measured for the optically, electrically, and simultaneously excited transient triplet absorption signals. For small driving currents, we find that the individual slopes do approximately add up to the simultaneous signal (as we found for the singlet signals), which implies that the triplet population is also not significantly affected by polaron or the electric field quenching under our experimental conditions. The absence of electric field quenching has also been recently shown by us using an alternative method [106].
In Figure 5, the experimentally measured triplet signals under various excitation intensities are plotted versus their corresponding singlet levels for both excitation modes. The plots are limited by the maximum laser excitation powers and device drive voltages that can be used. The graph shows that at the same singlet density about ten times more triplets are generated for electrical as compared to optical excitation. Critically, both datasets are well described by a linear function with slope of +1 in a double logarithmical plot which implies that both electrically and optically excited triplet densities depend linearly on the corresponding singlet densities. 
Thus, the singlet generation yield is a true constant and, in contrast to previous reports $[90,91]$, we find no electric field dependence with the drive voltages we have used. From these plots both $T^{\mathrm{el}} / S^{\mathrm{el}}=0.069 \pm 0.002$ and $T^{\mathrm{opt}} / S^{\mathrm{opt}}=0.0058 \pm$ 0.0002 for electrical and optical excitations, respectively, and consequently the el/opt ratio can be obtained, $11.9 \pm 0.8$.

In order to obtain an absolute singlet generation yield we need to know the absolute triplet formation yield for optical excitation, importantly, under the same experimental conditions, that is, low temperature and solid state. We have also developed a methodology for measuring the ISC yield in thin films by observation of the ground-state recovery of the photobleaching $[80,107]$. Using this method with subpicosecond time resolution and very low excitation doses, a yield of $\kappa^{\text {ISC }}=0.12 \pm 0.02$ is found for polyspirobifluorene in solid state at low temperature which compares very well with the fluorescence emission quantum yield of the polyspirobifluorene in solution, $0.82 \pm 0.03$. Given this, (3) yields an absolute singlet formation yield of $\chi=0.44 \pm 0.04$. It is clear that by successfully accounting for many possible experimental nonlinearities we still observe higher singlet exciton production than predicted by spin-independent charge recombination. Following a very similar methodology, Wallikewitz et al. [108] also find similar singlet generation ratios in other luminescent polymers. The question, thus, arises: do these results verify that charge recombination is spin dependent or are singlet excitons being generated in subsequent processes after the recombination step which give the false impression that more than $25 \%$ singlets are created directly from recombination.

To further expand on these results, we have made a series of measurements on different polymers and as a function of temperature. In Figure 6 is shown temperature-dependent results from the polymer poly $\left(9,9^{\prime}\right.$-dioctylfluorene-co-bis$\mathrm{N}, \mathrm{N}^{\prime}$ - (4-butylphenyl)-bis-N, $\mathrm{N}^{\prime}$-phenyl-1,4-phenylenediamine) (95:5 mol\%) (F8-PFB random copolymer). To accurately calculate the singlet yield from devices as a function of temperature, the effects on device performance as a function of temperature were first carefully characterized, and the electroluminescence output normalized with respect to drive current as a function of temperature. Clearly, it can be seen that at low temperature, $130 \mathrm{~K}$, one measures a far higher triplet population than at $250 \mathrm{~K}$, in the quasisteady state, as determined from the triplet induced absorption from a device. More importantly, a key measurement is the observation of the effect of temperature on the "delay electroluminescence (DE)" from the devices. As first shown by Sinha et al., there is a considerable, long-lived emission from devices after the drive current is turned off $[85,109]$. Spectrally resolving this DE shows that it has the same spectrum as the delayed emission seen with optical excitation arising from TF. As a function of temperature, it is found that this DE is very strong and long lived at $130 \mathrm{~K}$ but at room temperature it is quenched very rapidly. This correlates with the temperature-dependent triplet population measured in the devices (Figure 6). This general behaviour can be directly attributed to the temperature dependence of triplet exciton migration $[103,104,110]$, at high temperature the triplets can rapidly diffuse out of the recombination layer reducing triplet density and also find quenching sites before meeting another triplet to annihilate with. As will be shown in detail, from these results it is clear that the singlet yield will include a contribution from TF and that this will be strongly temperature dependent, arising from the temperature dependence of TTA not the charge recombination mechanism. In Figure 7 is given a graph of the measured temperature dependence of the singlet yield for F8-PFB-based devices. At low temperatures, we find a very high singlet yield which decreases as temperature increases and approaches ca. 0.35 at room temperature. Kondakov et al. [30, 63] have reported similar findings from small-molecule-based devices, again a strong DE signal is observed indicating a large contribution from TF to the overall singlet population in devices which clearly break the $25 \%$ singlet generation rule. Given that the recombination process does only produce $25 \%$ singlets, the results of Baldo et al. [60] can be reconciled with those of Kondakov et al. and ourselves.

\section{Is Charge Recombination Dependent on Spin?}

The evidence given above clearly indicates that TF contributes to the electroluminescence yield, but by how much and does this fit with the classical spin statistical production rate of singlets from TTA? In collaboration with Cambridge Display Technology, we have combined experimental measurement with detailed device modelling to put a quantitative measure on the contribution of TF to overall EL yield. We find at room temperature a voltage-dependent ( $\max 0.35$ ) TF contribution to the total EL signal [111] (Figure 8). We also show that as devices age, it is the TF contribution which is quenched causing the initial rapid reduction of EL yield before a stable plateau is reached when only the 0.25 singlets directly produced by recombination contribute to EL. Some questions have been raised about the outcoupling ratios assumed by Kondakov when calculating internal quantum efficiency, but certainly their small-molecule devices must be producing much more than 0.25 singlets as well.

\section{Historical Perspective on TTA}

The first observations of delayed fluorescence from triplettriplet annihilation in organic conjugated hydrocarbon solutions (including anthracene) were reported by Parker and Hatchard [23, 112]. Soon after, TF was also observed in molecular crystals of anthracene by Kepler et al. [22]. Jortner et al. developed a theory of singlet production during TTA using simple quantum mechanical spin statistics of two uncorrelated interacting triplet excitons to form an intermediate pair state postulated that nine possible spin configurations could result [113], as shown in Scheme 1. As a result of annihilation one excited singlet state is formed giving rise to delayed fluorescence for every 18 annihilating triplet excitons ( 9 pairs) giving a maximum singlet yield of 0.1 (accounting for triplet recycling; that is, the triplet states formed during TTA go through further annihilation steps 

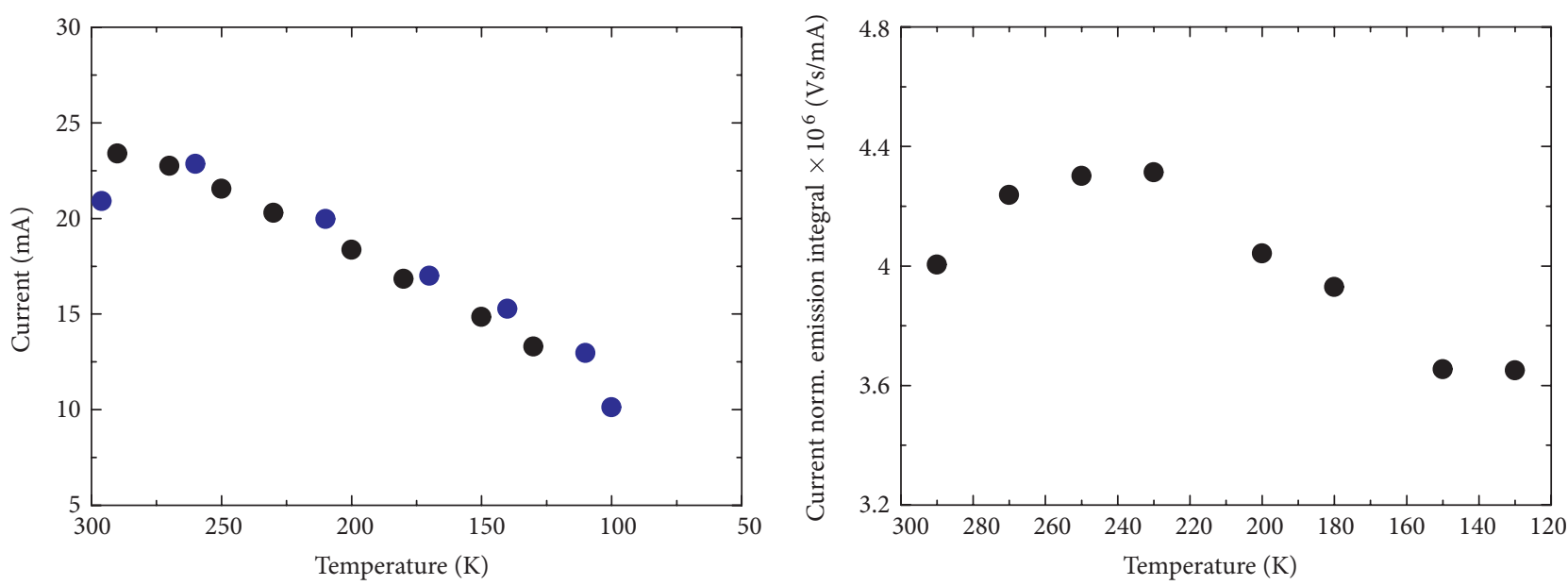

(a)
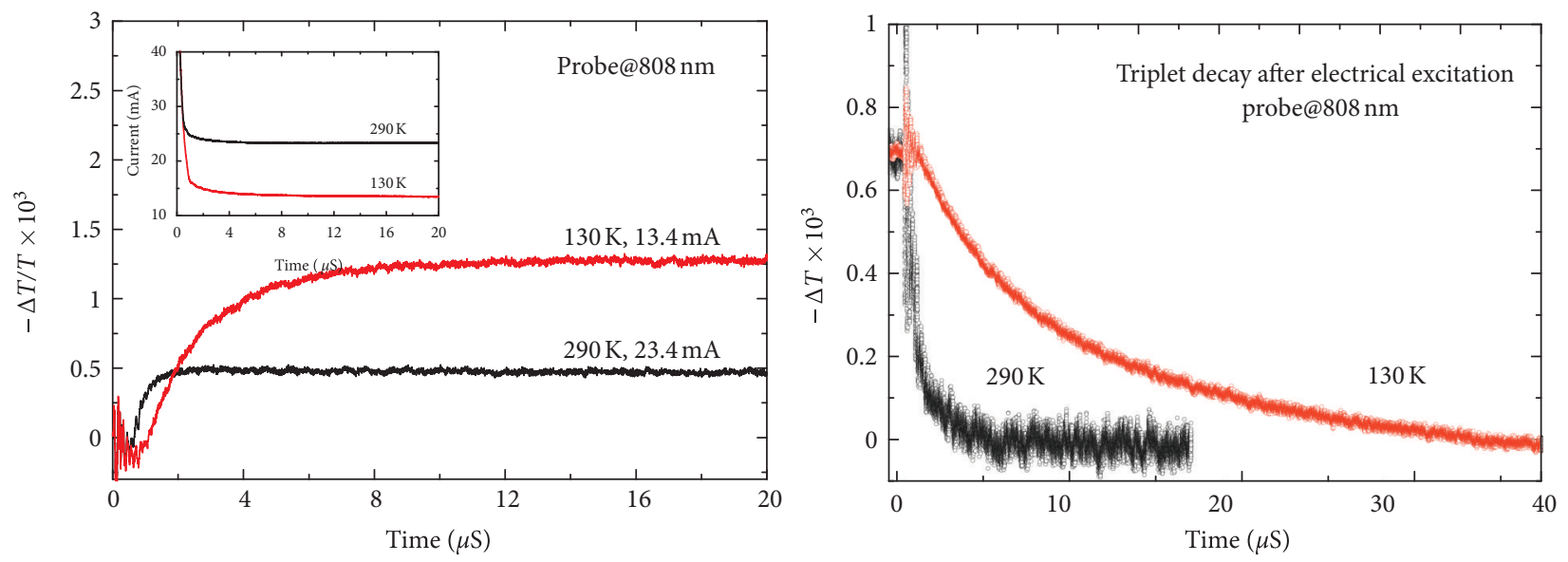

(b)

Figure 6: Data collected for the determination of the temperature dependence of the singlet generation yield. (a) Show how the electrical characteristics of the devices change as a function of temperature which enables the optical output from the devices to be scaled to take into account changes in the electrical characteristics. (b) Show how the triplet-population (measured by the triplet induced absorption) changes strongly with temperature, and also how the delayed electroluminescence is greatly quenched at high temperatures.

until all triplets are depleted). Experimentally, this purely theoretically scheme was questioned, mainly in the nature and decay channels of the quintuplet states. One can think of the intermediate pair (or encounter complex) as a highly excited excimer of various spin multiplicities in the ratio $1: 3: 5$. In this view, Birks explained delayed fluorescence from pyrene solutions $[24,114]$ as the result of the following channels: ${ }^{5}(\mathrm{AA})^{* *} \rightarrow{ }^{5}(\mathrm{AA})^{*} \rightarrow{ }^{1}(\mathrm{AA})^{*}$ and $\left({ }^{3} \mathrm{~A}^{*}+{ }^{1} \mathrm{~A}^{*}\right)$ the latter by a temperature-dependent disproportionation; ${ }^{3}(\mathrm{AA})^{* *} \rightarrow{ }^{1} \mathrm{~A}^{*}+{ }^{1} \mathrm{~A}$ and ${ }^{1}(\mathrm{AA})^{* *} \rightarrow{ }^{1}(\mathrm{AA})^{*}$, where $(\mathrm{AA})$ represents an excimer. Thus assuming the quintuplet yields 3 triplet states to 2 singlet states, then some $\sim 40 \%$ of triplets fuse yielding singlet states (note any triplet produced can go on to annihilate again until all triplets are used up given their very long monomolecular lifetime). Saltiel et al. [115, 116] made in-depth kinetic studies of TTA and proposed a modification of Birks' picture, whereby ${ }^{1}(\mathrm{AA})^{* *} \rightarrow{ }^{1}(\mathrm{AA})^{*}$; ${ }^{3}(\mathrm{AA})^{* *} \rightarrow{ }^{3} \mathrm{~A}^{*}+{ }^{1} \mathrm{~A} ;{ }^{5}(\mathrm{AA})^{* *} \rightarrow 1 / 10\left({ }^{1} \mathrm{~A}^{*}+{ }^{1} \mathrm{~A}\right)+9 / 10$ $\left({ }^{3} \mathrm{~A}^{*}+{ }^{3} \mathrm{~A}^{*}\right)$. Current work on the opposite process to TF, singlet fission (a singlet forming two triplets) which could be very important in solar cells; generating two excitons per photon to maximise charge production seems to indicate that a coherent superposition of singlet and triplet pair excited state wavefunctions are initially photocreated when $T_{n} \cong S_{1}$ such as in the case of tetracene and rubrene. Spin dipole-dipole interactions may then be responsible for forming the (TT) intermediate pair, which can again be thought of as an "excimer." Smith and Michl have written an excellent in-depth review on this subject [117]. A more simple and elegant argument though is that the quintets in most systems energetically cannot be created as the high energy quintuplet state cannot be formed with only twice the available triplet energy $[30,32]$. For $\mathrm{C}_{60}$, it has been calculated that a quintet state would physically break a C-C bond [118] and calculations for DPA, diphenylanthracene, indicate that the quintet state is too energetic to form from two triplets 


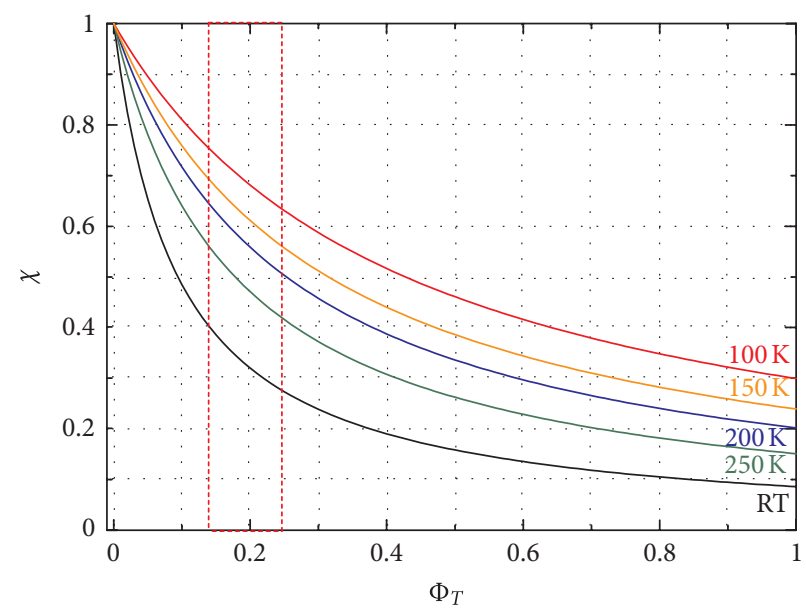

Figure 7: Calculated singlet yield for F8-PFB devices measured as a function of temperature. The two dashed red vertical lines give the upper and lower limits on our best determinations of the ISC yield of this polymer. Clearly, it is seen that the singlet yield depend strongly on temperature. At room temperature, the yield is ca. 0.33 , still appreciably above the 0.25 limit of spin-independent recombination.

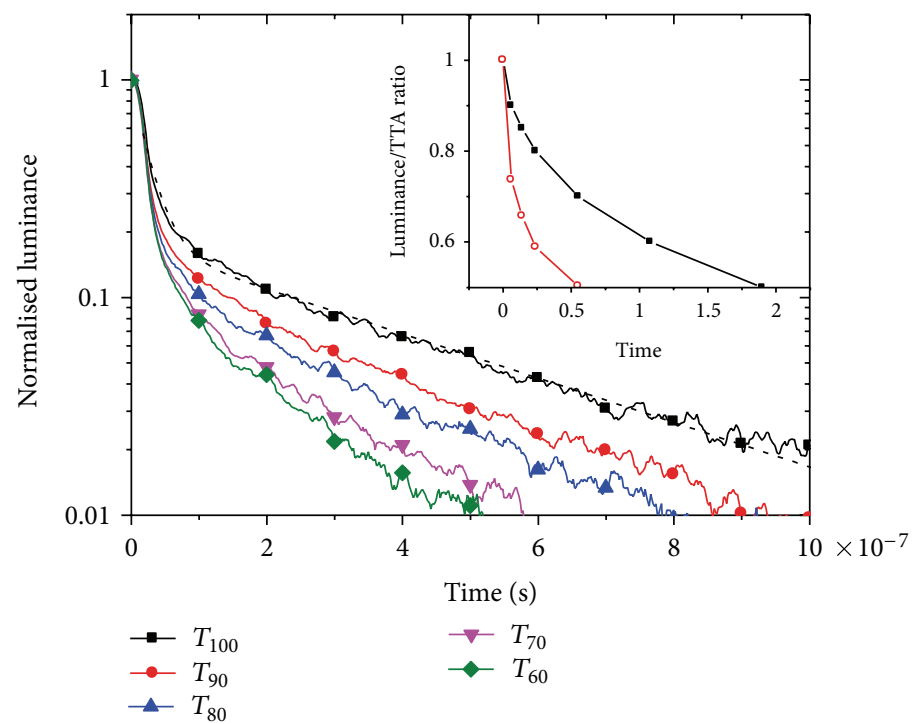

FIGURE 8: Delayed electroluminescence after glow as a function of device ageing showing both the 35\% contribution of delayed fluorescence to the total EL output and the loss of the DF with device age. $T_{60}$ implies that the devices has been run until its output has fallen to $60 \%$ of its initial light output.

[21]; in this scenario we would thus gain 0.2 singlets from TF as shown in Scheme 1. From Kondakov et al.s work on highly efficient fluorescent OLEDs using devices based on anthracene derivatives such as DPA as a host for blue fluorescence dyes ( $>0.95$ quantum yield) sandwiched between hole and electron transport layers, very simple devices, they demonstrated better than $8 \%$ external quantum efficiency for these devices. Calculating back, this implies an internal quantum yield of $>0.4$, way beyond the theoretical spinindependent recombination limit of 0.25 singlet generation. From studies of the DE from these devices they conclude that TF is contributing strongly $(\approx 0.2)$ to the total singlet yield in these devices. However, Kondakov et al. has made further claims of devices that exceed this value, indicating that TF in DPA approach levels of singlet generation that exceeds the 0.2 TTA singlet yield [30]. How is it possible to achieve higher singlet production yields than 0.2 from TTA? The energy diagram in Figure 9 shows schematically how this could be possible; on the right-hand side we have the situation where $2 E_{T_{1}}>E_{T_{n}}$ and $2 E_{T_{1}}>S_{1}$, in which case TTA can produce both $S_{1}$ and $T_{n}$ excited states. On the right we have a slightly different scenario, now $2 E_{T_{1}}>S_{1}$ but $2 E_{T_{1}}<E_{T_{n}}$ in this case it is now energetically not possible to form $T_{n}$ states, only $S_{1}$ states, giving a 0.5 singlet yield. In this case we could have in the best case a total ELQY $=0.25+(0.75 \times 0.5)=0.625$ with TF contributing $57 \%$ to the total EL. This then reaches 


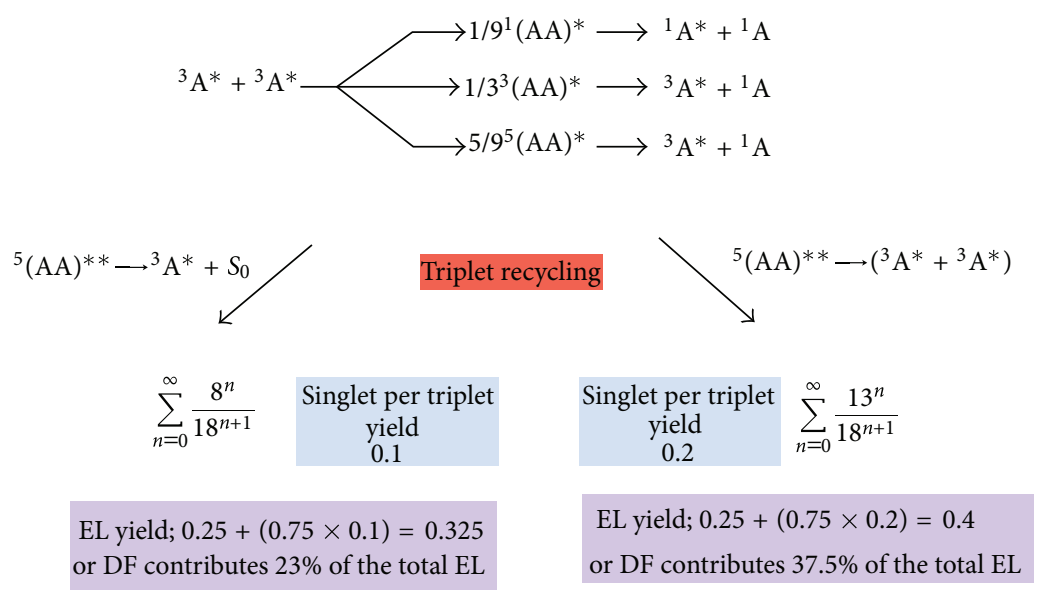

SCHEME 1: TTA decay channels.

into the realm of phosphorescent-based devices but from a fluorescent emitter, gaining all the benefits a fluorescent emitter gives to an OLED, especially in the blue. Therefore, there is a huge potential step change in OLED performance especially for blue emission in finding fluorescent emitters that fulfil this latter criterion.

In an attempt to reach this goal, Zhang and Forrest have revisited the potential of rubrene as an emitter which might yield 0.5 TF yield [33]. Rubrene has been studied for many years as an emissive material [34]; however it has an illdefined triplet energy in solid state as no phosphorescence has been observed, the long-time-delayed emission is dominated by DF, and so triplet energies are only known from pulsed radiolysis energy transfer measurements [119] with an error of order $\pm 0.2 \mathrm{eV}$. These measurements are made in solution and so gauging the degree to which the triplet energy relaxes in solid state is difficult [120]; therefore, in solid state the error on the triplet energy has to be of order $\pm 0.2-0.4 \mathrm{eV}$. Thus, it is not clear if $2 E_{T_{1}}=S_{1}$ in the solid state, yielding resonant singlet fission (SF) [121] or if $2 E_{T_{1}}<$ $E_{T_{n}}$ such that TTA can only proceed via the singlet decay channel to give $0.5 \mathrm{TF}$ yield. Zhang like Kondakov before [30] used a highly fluorescent dopant (1\%) in their rubrene devices which is populated by Forster transfer from the rubrene in an effort to avoid rubrene quenching by SF. These devices give $6.7 \% \mathrm{EQE}$ at low drive current, clearly breaking the $25 \%$ singlet generation rule. At higher currents, severe roll-off is observed which the authors attribute to onset of efficient singlet triplet annihilation (STA) [122]. However they also see the emergence of weak rubrene emission at high currents which suggests saturation of the dopant emitters [123] which may be the cause of the excessive STA. STA can be prevented by ensuring that Forster transfer from $S_{1}$ to $T_{1}$ (causing $T_{1}$ to $T_{n}$ absorption) cannot occur. A further complication with rubrene comes from the fact that $2 E_{T_{1}} \approx S_{1}$ causing the SF. If SF outcompetes the FRET to the fluorescent dopant, device efficiency will be reduced. Because of SF, the efficiency of pure rubrene devices is very poor. If the dopant sites saturate (especially at low doping levels), then SF will become an effective quenching mechanism; thus, there are many possible causes of loss of efficiency at high currents as shown in this work. The design rules for emitters for high TF devices become complexes when aiming for $0.5 \mathrm{TF}$ yield because of these problems associated with SF and STA.

Further support for this picture of device operation and the key role of TTA in enhancing the electroluminescent yield was given by Iwasaki et al. who have made an interesting observation on the role of triplet triplet annihilation [124]. From magnetic field dependencies of the TTA process, they conclude that in fact TTA contributes substantially to the final singlet yield. We have also shown that at room temperature, the typical triplet exciton lifetime reduces to below $250 \mathrm{~ns}$ compared to $>1 \mathrm{~s}$ at $10 \mathrm{~K}$ [125]; this in part is due to TTA but also other nonradiative pathways may become more efficient, this gives rise to the substantial temperature dependence of the ELQY we find in fluorescence devices as discussed above.

It is, thus, clear from this body of work that TTA via TF contributes substantially to device efficiency, and given optimal materials properties, fluorescent devices having $62.5 \%$ internal efficiency are theoretically possible. For this we require materials with triplet energies such that $2 E_{T_{1}}<$ $E_{T_{n}}$ with all triplets contributing to TTA, so reducing triplet mobility as much as possible by confinement with exciton blocking layers is important, as is the possible prevention of singlet fission by ensuring $2 E_{T_{1}} \neq S_{1}$. Last the photophysics of singlet triplet annihilation needs to be understood more clearly in order to design systems and device architectures that minimize this loss mechanism.

\section{Alternative Ways to Produce Singlet Excitons from Triplet States}

There are several known mechanisms for generating singlet emission long after all initially formed singlet states have decayed. Emission which involves triplet states can be categorised as either "P-type" or "E-type" emission. The former is singlet emission generated as a result of triplet fusion, as discussed above. E-type emission (or eosin-type) is a very different process, being a thermally activated long-lived 


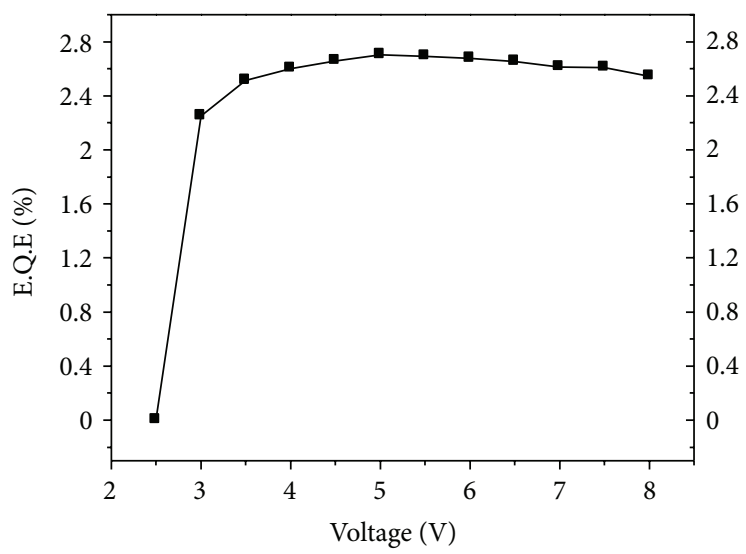

(a)

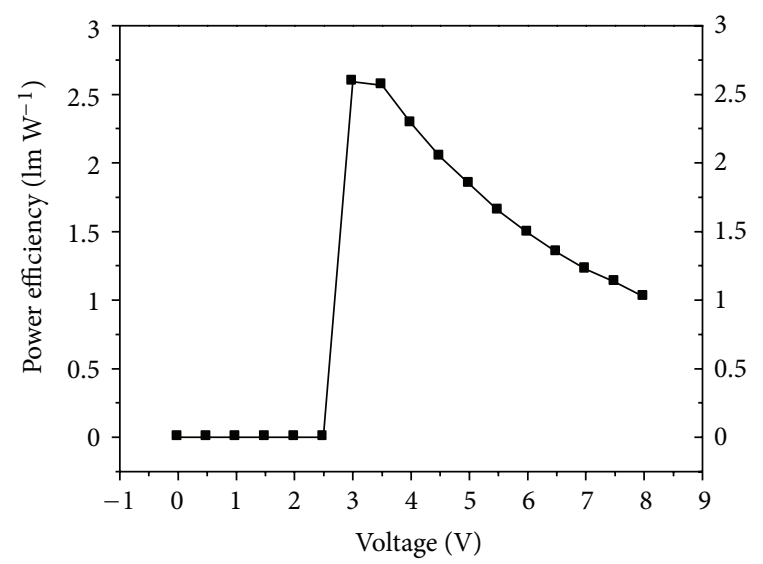

(c)

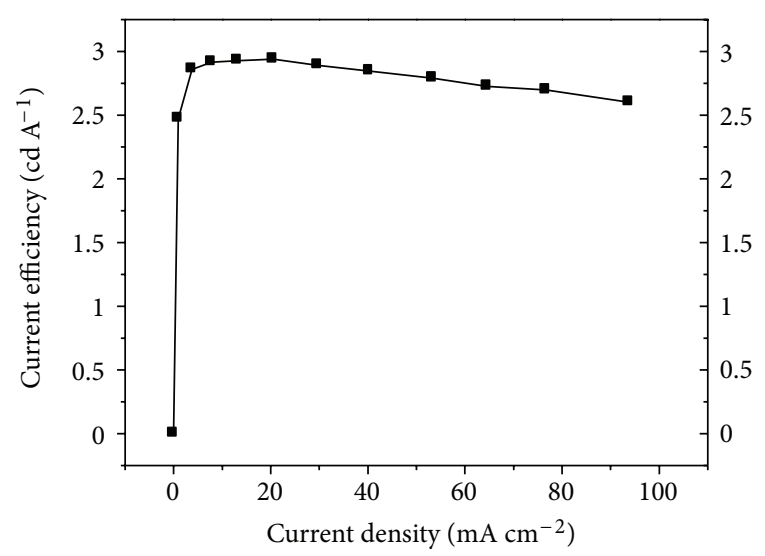

(b)

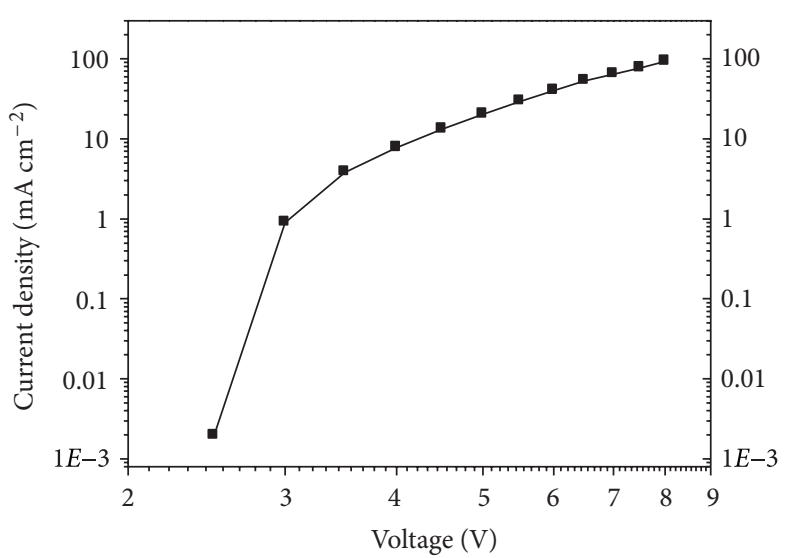

(d)

FIgure 9: PBD : TPBI (50:50 blend emitter layer) exciplex OLED device characteristics: EQE versus voltage (a), current efficiency versus current density (b), power efficiency versus voltage (c), and current density versus voltage (d).

emission, first observed by Perrin in 1929 [126]. It was not until Lewis and Kasha identified the triplet states of organic molecules that it was realised that the activation caused backcrossing of triplet states into the singlet manifold, that is, a process of thermal activation of a triplet state to a higher lying vibronic state followed by ISC to a resonant singlet vibronic state [127]. This state can then decay radiatively again with the normal fluorescence quantum yield. Since this is an activated process the rate is determined by a Boltzmanntype energy barrier proportional to $\exp \left(-\Delta E_{\mathrm{ST}} / k T\right)$, where $\Delta E_{\mathrm{ST}}$ is the singlet triplet energy gap, and the ISC rate. Thus, as $\Delta E_{\mathrm{ST}}$ decreases the rate of back transfer approaches the ISC rate. Given that for most materials the singlet fluorescence lifetime is many orders of magnitude shorter than the triplet lifetime, most triplet excitons will decay via the singlet channel as long as there is enough thermal activation, even with many recycling steps between singlet and triplet manifold. E-type emission also yields a common lifetime for both delayed emission and phosphorescence, where $k_{\mathrm{TA}}$ is the rate of thermally activated ISC. Hence at low temperature, phosphorescence dominates (radiative) triplet decay but at high temperature delayed singlet emission dominates. Theoretical modelling of the phosphine-supported $\mathrm{Cu}_{2}\left(\mu-\mathrm{NAr}_{2}\right)_{2}$ diamond core complex of Deaton et al. [128,
129] shows a very small exchange splitting of $750 \mathrm{~cm}^{-1}$ and clearly identifies the $\mathrm{HOMO}$ on the $\mathrm{Cu}_{2}-\mathrm{N}_{2}$ core with the LUMO on the peripheral aryl bringing units. The lowest excited state is found to comprise $>90 \%$ of this HOMOLUMO transition and hence nearly pure CT. This then yields a very small 2-electron exchange integral and the very low singlet triplet splitting required $[130,131]$. In terms of devices made form E-type emitters, one needs to have a high total luminescence quantum yield and a small $\Delta E_{\mathrm{ST}}$. The diamond core complex reported by Deaton yields green devices with external quantum efficiency of $16.1 \%$, close to that obtainable with Ir-based phosphors [128]. Further, there are also reports of all organic emitters which have low $\Delta E_{\mathrm{ST}}$ and appreciable E-type contribution [132]. Endo et al. have reported devices based on this mechanisms giving 5.3\% EQE in the blue/green $[133,134]$. Moreover, the key advantage here is increased stability of the (rigid) emitter, giving better device lifetime and more importantly potentially easier ways to obtain blue, since all the blue light is generated from the singlet state not the triplet. This avoids emitters with large HOMO-LUMO gaps which cause problems with electron or hole blocking (into the recombination layer); so fewer layers are required to match the emitter levels. This makes device design simpler, requiring fewer layers, increasing yield, and reducing cost. 
Negligible Stokes losses also improve device power efficiency because electrical energy is not wasted, converting singlets to triplets, as in phosphorescence, losing some 0.3 to $0.7 \mathrm{~V}$. This is very important for maintaining high $\mathrm{lm} / \mathrm{W}$ values. Thus, using E-type DF has many advantages over phosphorescence, but requires the special criterion of very small singlet triplet gap, but most importantly this can give devices with a theoretical yield of $100 \%$ ! This triplet harvesting paradigm is essentially unexplored in OLED research but can give a real step change in both performance and lifetime.

Endo's original work used an emitter that had strong internal charge transfer character to produce very low electron exchange energy, the natural extension of this is the intermolecular exciplex. An exciplex is an intermolecular charge-transfer state formed under photo- or electrical excitation by the interaction of an electron donor (D) and an electron acceptor (A) [135]. The wavelength of exciplex emission depends on the ionisation potential and electron affinity of the donor and acceptor molecules, respectively. There is literature dating back to the early days of OLED research discussing the pros, but mostly the cons, of exciplexes [136]. This early work focused on exciplexes formed unintentionally at the interface between a transport layer and the emitter layer, usually seen only in electroluminescence (EL) and not in photoluminescence (PL). The first report of interfacial exciplex emission was in 1998 by Itano et al. [137] and then in a blended exciplex device by Cocchi et al. [138]. The latter devices were inefficient as they incorporated the emitter molecules in a polycarbonate matrix and the exciplex had low photoluminescence quantum yield (PLQY) $\left(\Phi_{\mathrm{PL}} 17 \%\right)$. The authors clearly described exciplex evolution from a tightly bound $|\mathbf{D A}\rangle^{*}$ exciplex to an ionic $\left|\mathbf{D}^{+} \mathbf{A}^{-}\right\rangle^{*}$ ion pair, and the effect of Coulomb relaxation which yields large red shifts, thereby explaining the previously observed "electroplex" [139]. Palilis et al. [140] were the first to report true blend devices, using an exciplex system of high PLQY $\left(\Phi_{\mathrm{PL}} 62 \%\right)$ between a triarylamine hole transporter (the $\mathbf{D}$ unit) and a highly fluorescent $\left(\Phi_{\mathrm{PL}} 85 \%\right)$ silole-based emitter and electron transporter (the $\mathbf{A}$ unit). Devices with external quantum efficiency (EQE) of 3.4\% were reported, which at the time was excellent. These results clearly show that it is possible to engineer exciplexes with strong ground-state coupling and thus high luminescence efficiency.

Usually E-type emission is an inefficient process as most materials have large electron exchange energies. Frederichs and Staerk [141] were the first to show experimentally the assertion of Beens and Weller [142] that thermally assisted ISC from an exciplex triplet to singlet manifold can occur, and that certain exciplexes have very small exchange energies $(<0.1 \mathrm{eV})$ with clear E-type emission from the exciplex. They also showed the importance of coupling to the $\mathbf{D}$ ground state to achieve high luminescence yields. These solution studies also showed the role of environment polarity in stabilising the degree of charge separation in the exciplex. Only for highly polar environments is the radical ion pair stabilised. Given that in the solid state the environment will be only weakly or moderately polar there will be a driving force to stabilise the more excitonic-like $|\mathbf{D A}\rangle^{*}$ exciplex rather than the full ion pair $\mid \mathbf{D}^{+} \mathbf{A}^{-}>^{*}$. This will have the benefits of enhancing both the ground-state coupling and the luminescence yields and will limit the electric field quenching of the exciplex in the device. The importance in the context of OLEDs of the fact that, in principle, exciplexes can have vanishingly small exchange energies [143] was first highlighted by Cocchi et al. in 2006 [144] who discussed the possibilities of electrophosphorescence from exciplexes. However, their system (donor TPD : acceptor BCP in a polycarbonate matrix) has a rather large singlet-triplet gap, $\Delta E c a .0 .4 \mathrm{eV}$. More interesting, however, is the fact that in this system the electron-hole pair energy, $E_{h}=I_{D}-A_{A}$, is less than that required to form an excited donor singlet. However, as shown by Morteani et al. [145] direct injection into the exciplex is possible and this gives the benefit of low drive voltages, which is a critical finding.

The first report of an intentional exciplex-based device giving E-type exciplex emission was by Goushi et al. in 2012 [146]. The donor molecule is a triarylamine and the acceptor a triarylborane derivative, these were coevaporated in a 50:50 blend emission layer. OLEDs with EQE of 5.4\% were realised from an exciplex system having an PLQY of only $26 \%$, indicating that far more than $25 \%$ singlets were being generated in the device. Subsequently, Goushi has reported a device giving up to $10 \% \mathrm{EQE}, 47 \mathrm{~lm} \mathrm{~W}^{-1}$ for green emission [147]. The device structures in both types of device are extremely simple, consisting of only three organic layers, an HTL of the donor, the 50:50 emitter layer, and an ETL layer consisting of the acceptor. A very important added benefit of such a very simple device structure is that it gives a very low working voltages, $c a$. $2.5 \mathrm{~V}$. This is vitally important for high luminance efficacy lighting and good compatibility with CMOS backplanes in mobile devices. The high EQE and luminance power efficiencies derive from the efficient direct electron-hole capture at the exciplex. There are no voltage drops associated with charge injection and transport through additional layers, and the usual necessity of forcing the electron and hole onto a single molecular emitter site is overcome [145]. Thus, E-type exciplex devices have many advantages over phosphorescence based devices, notably a very simple device structure (two materials in three layers) and very high power efficiency. In Figure 9, results from a deep blue exciplex device (structure given in Figure 10) based on PBD (D) and TPBI (A), show far higher than $25 \%$ total singlet production in electroluminescence. The exciplex has a PLQY of $26 \%$ and EQE of $2.6 \%$ (unoptimized) at $450 \mathrm{~nm}$. For this low PLQY of the emitter, the theoretical maximum device EQE is 1.3\% [148]. However, in this exciplex system the D (NPB) has a triplet level [149] lower than that of the exciplex triplet which we believe strongly quenches the exciplex triplet state, but the subsequent high triplet population gives rise to strong TF which enhances the device efficiency. This quenching route can be overcome by careful materials design, making sure that both the $\mathbf{D}$ and $\mathbf{A}$ have higher triplet levels than the exciplex. Bittner et al. [150] calculated for a mixed TFB:F8BT monomer system that this backtransfer mechanism is possible; however, they assumed thermally activated singlet transfer, in line with much of the work from Morteani et al. [151] on exciton regeneration at 


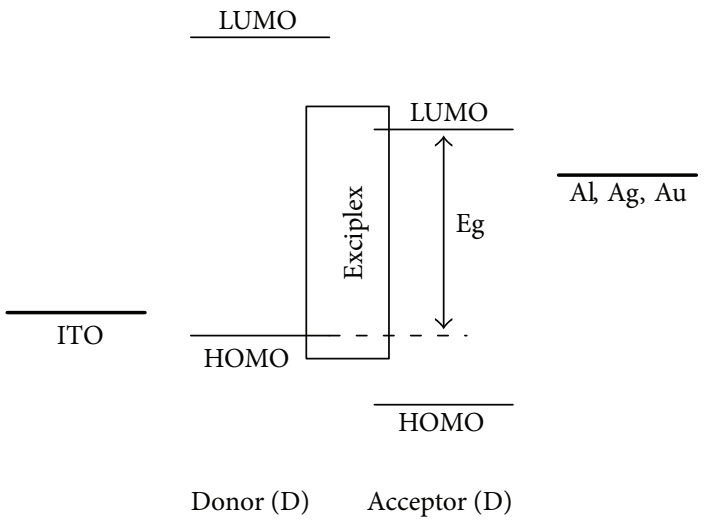

FIGURE 10: Schematic energy level diagram for a simple low turn on volatge exciplex OLED. Comparing to the device used in Figure 9, the donor layer is PBD the acceptor layer is TPBI and the emitter layer a 50:50 blend of NPB and TPBI. Here the NPB is a good hole transport layer and TPBI is a good electron transport layer. Layers are deposited by vacuum sublimation.

interfaces. This behaviour can also readily be explained as backtransfer via the triplet channel and that the regenerated excitons arise from TF within the $\mathbf{D}$ manifold.

Polymer-based exciplex systems have been reported [152, 153] but they have an extra complexity in that many exciplex systems give rise to efficient charge production, as used in photovoltaic applications. As opposed to the polymeric TFB:F8BT system which can yield OLEDs with $>19 \mathrm{~lm} \mathrm{~W}^{-1}$, PFB:F8BT (PFB has one extra phenylamine unit per polymer repeat unit than TFB) efficiently forms charge pairs with no emission, but also has a high triplet exciton yield [54]. This could indicate differences in exciplex stabilisation if an ion pair is the initial species created by photoexcitation, as fielddependent quenching would suggest [151]. The result implies rapid singlet exciplex ionisation as well as quenching of the triplet exciplex to the triplet state of the PFB. The PFB:F8BT exciplex has the larger charge separation radius, leading to greater ion pair character in the exciplex.

E-type emission, thus, offers great potential for highly power-efficient fluorescent OLEDs, ideal for both lighting applications and micro-OLEDs fabricated on CMOS chips. Compared to devices exploiting TF, E-type emitters offer a theoretical maximum $100 \%$ internal efficiency without the drawback of requiring a high triplet host material, the problem which bedevils high efficiency blue phosphorescent emitters. Moreover, they allow very simple device architecture to be used whilst maintaining extremely high optical and electrical efficiency. This is very important for largearea lighting applications and the use of solution processing. "Exciplex blend" devices, thus, opens a new chapter in OLED devices, one which has great promise for many applications requiring large area or high yield fabrication.

\section{References}

[1] G. He, C. Rothe, S. Murano, A. Werner, O. Zeika, and J. Birnstock, "White stacked OLED with $38 \mathrm{~lm} / \mathrm{W}$ and 100,000-hour lifetime at $1000 \mathrm{~cd} / \mathrm{m} 2$ for display and lighting applications," Journal of the Society for Information Display, vol. 17, no. 2, pp. 159-165, 2009.
[2] N. Ide, H. Tsuji, N. Ito, Y. Matsuhisa, S. Houzumi, and T. Nishimori, "White OLED devices and processes for lighting applications," in Organic Photonics Iv., P. L. Heremans, R. Coehoorn, and C. Adachi, Eds., vol. 7722, Spie-Int Soc Optical Engineering, Bellingham, Wash, USA, 2010.

[3] Y. S. Tyan, Y. Q. Rao, X. F. Ren et al., Tandem Hybrid White OLED Devices With Improved Light Extraction, Campbell: Society For Information Display, 2009.

[4] C. Adachi, R. C. Kwong, P. Djurovich et al., "Endothermic energy transfer: a mechanism for generating very efficient highenergy phosphorescent emission in organic materials," Applied Physics Letters, vol. 79, no. 13, pp. 2082-2084, 2001.

[5] V. Sivasubramaniam, F. Brodkorb, S. Hanning et al., "Fluorine cleavage of the light blue heteroleptic triplet emitter FIrpic," Journal of Fluorine Chemistry, vol. 130, no. 7, pp. 640-649, 2009.

[6] V. Sivasubramaniam, F. Brodkorb, S. Hanning et al., "Investigation of FIrpic in PhOLEDs via LC/MS technique," Central European Journal of Chemistry, vol. 7, no. 4, pp. 836-845, 2009.

[7] K. T. Kamtekar, A. P. Monkman, and M. R. Bryce, "Recent advances in white organic light-emitting materials and devices (WOLEDS)," Advanced Materials, vol. 22, no. 5, pp. 572-582, 2010.

[8] A. Van Dijken, J. J. A. M. Bastiaansen, N. M. M. Kiggen et al., "Carbazole compounds as host materials for triplet emitters in organic light-emitting diodes: polymer hosts for high-efficiency light-emitting diodes," Journal of the American Chemical Society, vol. 126, no. 24, pp. 7718-7727, 2004.

[9] K. Brunner, A. Van Dijken, H. Börner, J. J. A. M. Bastiaansen, N. M. M. Kiggen, and B. M. W. Langeveld, "Carbazole compounds as host materials for triplet emitters in organic light-emitting diodes: tuning the HOMO level without influencing the triplet energy in small molecules," Journal of the American Chemical Society, vol. 126, no. 19, pp. 6035-6042, 2004.

[10] Y. C. Chen, G. S. Huang, C. C. Hsiao, and S. A. Chen, "High triplet energy polymer as host for electrophosphorescence with high efficiency," Journal of the American Chemical Society, vol. 128, no. 26, pp. 8549-8558, 2006.

[11] S. O. Jeon, K. S. Yook, C. W. Joo, and J. Y. Lee, "High-efficiency deep-blue-phosphorescent organic light-emitting diodes using a phosphine oxide and a phosphine sulfide high-tripletenergy host material with bipolar charge-transport properties," Advanced Materials, vol. 22, no. 16, pp. 1872-1876, 2010. 
[12] H. Sasabe, J. I. Takamatsu, T. Motoyama et al., "High-efficiency blue and white organic light-emitting devices incorporating a blue iridium carbene complex," Advanced Materials, vol. 22, no. 44, pp. 5003-5007, 2010.

[13] H. A. Al-Attar, G. C. Griffiths, T. N. Moore et al., "Highly efficient, solution-processed, single-layer, electrophosphorescent diodes and the effect of molecular dipole moment," Advanced Functional Materials, vol. 21, no. 12, pp. 2376-2382, 2011.

[14] H. A. Al-Attar and A. P. Monkman, "Erratum: solution processed multilayer polymer light-emitting diodes based on different molecular weight host (Journal of Applied Physics (2011) 109 (074516))," Journal of Applied Physics, vol. 110, no. 2, Article ID 029905, 2011.

[15] N. Tian, D. Lenkeit, S. Pelz et al., "Screening structure-property correlations and device performance of $\operatorname{Ir}$ (III) complexes in multi-layer PhOLEDs," Dalton Transactions, vol. 40, pp. 1162911635, 2011.

[16] K. S. Yook and J. Y. Lee, "Solution processed multilayer deep blue and white phosphorescent organic light-emitting diodes using an alcohol soluble bipolar host and phosphorescent dopant materials," Journal of Materials Chemistry, vol. 22, pp. 14546-14550, 2012.

[17] J. S. Kim, R. H. Friend, I. Grizzi, and J. H. Burroughes, “Spin-cast thin semiconducting polymer interlayer for improving device efficiency of polymer light-emitting diodes," Applied Physics Letters, vol. 87, no. 2, pp. 1-3, 2005.

[18] X. Gong, S. Wang, D. Moses, G. C. Bazan, and A. J. Heeger, "Multilayer polymer light-emitting diodes: white-light emission with high efficiency," Advanced Materials, vol. 17, no. 17, pp. 2053-2058, 2005.

[19] Y. Sun, N. C. Giebink, H. Kanno, B. Ma, M. E. Thompson, and S. R. Forrest, "Management of singlet and triplet excitons for efficient white organic light-emitting devices," Nature, vol. 440, no. 7086, pp. 908-912, 2006.

[20] S. Reineke, F. Lindner, G. Schwartz et al., "White organic lightemitting diodes with fluorescent tube efficiency," Nature, vol. 459, no. 7244, pp. 234-238, 2009.

[21] M. E. Kondakova, J. C. Deaton, T. D. Pawlik et al., "Highly efficient fluorescent-phosphorescent triplet-harvesting hybrid organic light-emitting diodes," Journal of Applied Physics, vol. 107, no. 1, Article ID 014515, 2010.

[22] R. G. Kepler, J. C. Caris, P. Avakian, and E. Abramson, “Triplet excitons and delayed fluorescence in anthracene crystals," Physical Review Letters, vol. 10, no. 9, pp. 400-402, 1963.

[23] C. A. Parker and C. G. Hatchard, "Delayed fluorescence from solutions of anthracene and phenanthrene," in Proceedings of the Royal Society of London Series a-Mathematical and Physical Sciences, vol. 269, p. 574, 1962.

[24] J. B. Birks, "On the delayed fluorescence of pyrene solutions," Journal of Physical Chemistry, vol. 67, no. 10, pp. 2199-2200, 1963.

[25] R. P. Groff, R. E. Merrifield, and P. Avakian, "Singlet and triplet channels for triplet-exciton fusion in anthracene crystals," Chemical Physics Letters, vol. 5, no. 3, pp. 168-170, 1970.

[26] M. Pope, Geacinto. Ne, and F. Vogel, "Singlet exciton fission and triplet-triplet exciton fusion in crystalline tetracene," Molecular Crystals and Liquid Crystals, vol. 6, p. 83, 1969.

[27] J. Jortner, S. A. Rice, J. L. Katz, and S. I. L. Choi, "Triplet excitons in crystals of aromatic molecules," The Journal of Chemical Physics, vol. 42, no. 1, pp. 309-323, 1965.
[28] R. S. Knox and C. E. Swenberg, "Direct radiative Excitonexciton annihilation," The Journal of Chemical Physics, vol. 44, no. 7, pp. 2577-2580, 1966.

[29] C. E. Swenberg, "Theory of triplet exciton annihilation in polyacene crystals," The Journal of Chemical Physics, vol. 51, no. 5, pp. 1753-1764, 1969.

[30] D. Y. Kondakov, T. D. Pawlik, T. K. Hatwar, and J. P. Spindler, "Triplet annihilation exceeding spin statistical limit in highly efficient fluorescent organic light-emitting diodes," Journal of Applied Physics, vol. 106, no. 12, Article ID 124510, 2009.

[31] B. Dick, "AM1 and INDO/S calculations on electronic singlet and triplet states involved in excited-state intramolecular proton transfer of 3-hydroxyflavone," Journal of Physical Chemistry, vol. 94, no. 15, pp. 5752-5756, 1990.

[32] B. Dick and B. Nickel, "Accessibility of the lowest quintet state of organic molecules through triplet-triplet annihilation; an indo ci study," Chemical Physics, vol. 78, no. 1, pp. 1-16, 1983.

[33] Y. Zhang and S. R. Forrest, "Triplets Contribute to Both an Increase and Loss in Fluorescent Yield in Organic Light Emitting Diodes," Physical Review Letters, vol. 108, Article ID 267404, 5 pages, 2012.

[34] R. W. T. Higgins, A. P. Monkman, H. G. Nothofer, and U. Scherf, "Effects of singlet and triplet energy transfer to molecular dopants in polymer light-emitting diodes and their usefulness in chromaticity tuning," Applied Physics Letters, vol. 79, no. 6, pp. 857-859, 2001.

[35] A. Köhler and H. Bässler, "Triplet states in organic semiconductors," Materials Science and Engineering R, vol. 66, no. 4-6, pp. 71-109, 2009.

[36] A. P. Monkman, H. D. Burrows, M. D. Miguel, I. Hamblett, and S. Navaratnam, "Measurement of the S0-T1 energy gap in poly (2-methoxy,5-( $2^{\prime}$-ethyl-hexoxy)-p-phenylenevinylene) by triplet-triplet energy transfer," Chemical Physics Letters, vol. 307, no. 5-6, pp. 303-309, 1999.

[37] A. P. Monkman, H. D. Burrows, L. J. Hartwell, L. E. Horsburgh, I. Hamblett, and S. Navaratnam, "Triplet energies of $\pi$ conjugated polymers," Physical Review Letters, vol. 86, no. 7, pp. 1358-1361, 2001.

[38] M. Knupfer, "Exciton binding energies in organic semiconductors," Applied Physics A, vol. 77, no. 5, pp. 623-626, 2003.

[39] S. F. Alvarado, P. F. Seidler, D. G. Lidzey, and D. D. C. Bradley, "Direct determination of the exciton binding energy of conjugated polymers using a scanning tunneling microscope," Physical Review Letters, vol. 81, no. 5, pp. 1082-1085, 1998.

[40] M. Rohlfing and S. G. Louie, "Optical Excitations in Conjugated Polymers," Physical Review Letters, vol. 82, no. 9, pp. 1959-1962, 1999.

[41] S. M. King, H. L. Vaughan, and A. P. Monkman, "Orientation of triplet and singlet transition dipole moments in polyfluorene, studied by polarised spectroscopies," Chemical Physics Letters, vol. 440, no. 4-6, pp. 268-272, 2007.

[42] A. Monkman and H. D. Burrows, "Backbone planarity effects on triplet energies and electron-electron correlation in luminescent conjugated polymers," Synthetic Metals, vol. 141, no. 1-2, pp. 81-86, 2004.

[43] A. P. Monkman, H. D. Burrows, I. Hamblett, S. Navarathnam, M. Svensson, and M. R. Andersson, "The effect of conjugation length on triplet energies, electron delocalization and electronelectron correlation in soluble polythiophenes," Journal of Chemical Physics, vol. 115, no. 19, pp. 9046-9049, 2001. 
[44] S. King, C. Rothe, and A. Monkman, "Triplet build in and decay of isolated polyspirobifluorene chains in dilute solution," Journal of Chemical Physics, vol. 121, no. 21, pp. 10803-10808, 2004.

[45] J. S. De Melo, H. D. Burrows, M. Svensson, M. R. Andersson, and A. P. Monkman, "Photophysics of thiophene based polymers in solution: the role of nonradiative decay processes," Journal of Chemical Physics, vol. 118, no. 3, pp. 1550-1556, 2003.

[46] S. M. King, R. Matheson, F. B. Dias, and A. P. Monkman, "Enhanced triplet formation by twisted intramolecular chargetransfer excited states in conjugated oligomers and polymers," Journal of Physical Chemistry B, vol. 112, no. 27, pp. 8010-8016, 2008.

[47] Z. H. Kafafi, Organic Electroluminescence, Marcel Dekker, New York, NY, USA, 2005.

[48] J. Kalinowski, L. C. Palilis, W. H. Kim, and Z. H. Kafafi, "Determination of the width of the carrier recombination zone in organic light-emitting diodes," Journal of Applied Physics, vol. 94, no. 12, pp. 7764-7767, 2003.

[49] C. Rothe, H. A. Al Attar, and A. P. Monkman, "Absolute measurements of the triplet-triplet annihilation rate and the chargecarrier recombination layer thickness in working polymer lightemitting diodes based on polyspirobifluorene," Physical Review $B$, vol. 72, no. 15, Article ID 155330, 9 pages, 2005.

[50] W. Barford, "Theory of singlet exciton yield in light-emitting polymers," Physical Review B, vol. 70, no. 20, Article ID 205204, 8 pages, 2004.

[51] M. Reufer, M. J. Walter, P. G. Lagoudakis et al., "Spin-conserving carrier recombination in conjugated polymers," Nature Materials, vol. 4, no. 4, pp. 340-346, 2005.

[52] S. Karabunarliev and E. R. Bittner, "Spin-dependent electronhole capture kinetics in luminescent conjugated polymers," Physical Review Letters, vol. 90, no. 5, Article ID 057402, 4 pages, 2003.

[53] M. Segal, M. Singh, K. Rivoire, S. Difley, T. Van Voorhis, and M. A. Baldo, "Extrafluorescent electroluminescence in organic light-emitting devices," Nature Materials, vol. 6, no. 5, pp. 374378, 2007.

[54] T. A. Ford, H. Ohkita, S. Cook, J. R. Durrant, and N. C. Greenham, "Direct observation of intersystem crossing in charge-pair states in polyfluorene polymer blends," Chemical Physics Letters, vol. 454, no. 4-6, pp. 237-241, 2008.

[55] M. Wohlgenannt, K. Tandon, S. Mazumdar, S. Ramasesha, and Z. V. Vardeny, "Formation cross-sections of singlet and triplet excitons in $\pi$-conjugated polymers," Nature, vol. 409, no. 6819 , pp. 494-497, 2001.

[56] J. S. Kim, P. K. H. Ho, N. C. Greenham, and R. H. Friend, "Electroluminescence emission pattern of organic light-emitting diodes: implications for device efficiency calculations," Journal of Applied Physics, vol. 88, no. 2, pp. 1073-1081, 2000.

[57] C. Rothe, S. M. King, and A. P. Monkman, "Direct measurement of the singlet generation yield in polymer light-emitting diodes," Physical Review Letters, vol. 97, no. 7, Article ID 076602, 2006.

[58] A. P. Monkman, C. Rothe, and S. M. King, "Singlet generation yields in organic light-emitting diodes," Proceedings of the IEEE, vol. 97, no. 9, pp. 1597-1605, 2009.

[59] L. C. Lin, H. F. Meng, J. T. Shy et al., "Triplet-to-singlet exciton formation in poly(p-phenylene-vinylene) light-emitting diodes," Physical Review Letters, vol. 90, no. 3, Article ID 036601, 4 pages, 2003.
[60] M. A. Baldo, D. F. O’Brien, M. E. Thompson, and S. R. Forrest, "Excitonic singlet-triplet ratio in a semiconducting organic thin film," Physical Review B, vol. 60, no. 20, pp. 14422-14428, 1999.

[61] M. Segal, M. A. Baldo, R. J. Holmes, S. R. Forrest, and Z. G. Soos, "Excitonic singlet-triplet ratios in molecular and polymeric organic materials," Physical Review B, vol. 68, no. 7, Article ID 075211, 14 pages, 2003.

[62] D. Y. Kondakov, "Role of triplet-triplet annihilation in highly efficient fluorescent devices," Journal of The Society for Information Display, vol. 17, no. 2, pp. 137-144.

[63] D. Y. Kondakov, "Characterization of triplet-triplet annihilation in organic light-emitting diodes based on anthracene derivatives," Journal of Applied Physics, vol. 102, no. 11, Article ID 114504, 5 pages, 2007.

[64] K. Tandon, S. Ramasesha, and S. Mazumdar, "Electron correlation effects in electron-hole recombination in organic lightemitting diodes," Physical Review B, vol. 67, no. 4, Article ID 045109, 19 pages, 2003.

[65] M. Wohlgenannt, X. M. Jiang, Z. V. Vardeny, and R. A. J. Janssen, "Conjugation-length dependence of spin-dependent exciton formation rates in $\Pi$-conjugated oligomers and polymers," Physical Review Letters, vol. 88, no. 19, pp. 1974011-1974014, 2002.

[66] F. Feller and A. P. Monkman, "Electroabsorption studies of poly(2,5-pyridinediyl)," Physical Review B, vol. 60, no. 11, pp. 8111-8116, 1999.

[67] W. T. Simpson, "Resonance force theory of carotenoid pigments," Journal of the American Chemical Society, vol. 77, pp. 6164-6168, 1955.

[68] W. T. Simpson, "Internal dispersion forces. The polyenes," Journal of the American Chemical Society, vol. 73, no. 11, pp. 5363-5367, 1951.

[69] E. W. Snedden, A. P. Monkman, and F. B. Dias, "Photophysics of charge generation in organic photovoltaic materials: kinetic studies of geminate and free polarons in a model donor/acceptor system," Journal of Physical Chemistry C, vol. 116, pp. 86-97, 2012.

[70] E. W. Snedden, A. P. Monkman, and F. B. Dias, "Kinetic studies of geminate polaron pair recombination, dissociation, and efficient triplet exciton formation in PC:PCBM organic photovoltaic blends," Journal of Physical Chemistry C, vol. 116, pp. 4390-4398, 2012.

[71] S. Karabunarliev and E. R. Bittner, "Dissipative dynamics of spin-dependent electron-hole capture in conjugated polymers," Journal of Chemical Physics, vol. 119, no. 7, pp. 3988-3995, 2003.

[72] V. Jankus, C. Winscom, and A. P. Monkman, “The photophysics of singlet, triplet, and degradation trap states in 4,4- N, N' dicarbazolyl- 1, 1' -biphenyl," Journal of Chemical Physics, vol. 130, no. 7, Article ID 074501, 2009.

[73] V. Jankus, C. Winscom, and A. P. Monkman, "Critical role of triplet exciton interface trap states in bilayer films of NPB and Ir(piq)3," Advanced Functional Materials, vol. 21, no. 13, pp. 2522-2526, 2011.

[74] S. Yin, L. Chen, P. Xuan, K. Q. Chen, and Z. Shuai, "Field effect on the singlet and triplet exciton formation in organic/polymeric light-emitting diodes," Journal of Physical Chemistry B, vol. 108, no. 28, pp. 9608-9613, 2004.

[75] M. Das, S. Ramasesha, and S. Mazumdar, "Role of electronelectron interactions on spin effects in electron-hole recombination in organic light emitting diodes," Synthetic Metals, vol. 155, no. 2, pp. 270-273, 2005. 
[76] S. Difley, D. Beljonne, and T. V. Voorhis, "On the singlet-triplet splitting of geminate electron-hole pairs in organic semiconductors," Journal of the American Chemical Society, vol. 130, no. 11, pp. 3420-3427, 2008.

[77] D. Beljonne, Z. Shuai, A. Ye, and J. L. Brédas, "Chargerecombination processes in oligomer- and polymer-based lightemitting diodes: a molecular picture," Journal of the Society for Information Display, vol. 13, no. 5, pp. 419-427, 2005.

[78] L. Chen, L. Zhu, and Z. Shuai, "Singlet-triplet splittings and their relevance to the spin-dependent exciton formation in light-emitting polymers: an EOM/CCSD study," Journal of Physical Chemistry A, vol. 110, no. 50, pp. 13349-13354, 2006.

[79] M. Gordon and W. R. Ware, Eds., The Exciplex, Academic Press, New York, NY, USA, 1975.

[80] S. M. King, C. Rothe, D. Dai, and A. P. Monkman, "Femtosecond ground state recovery: measuring the intersystem crossing yield of polyspirobifluorene," Journal of Chemical Physics, vol. 124, no. 23, Article ID 234903, 2006.

[81] M. K. Lee, M. Segal, Z. G. Soos, J. Shinar, and M. A. Baldo, "Yield of singlet excitons in organic light-emitting devices: a double modulation photoluminescence-detected magnetic resonance study," Physical Review Letters, vol. 94, no. 13, Article ID 137403, 2005.

[82] M. Segal, M. A. Baldo, M. K. Lee, J. Shinar, and Z. G. Soos, "Frequency response and origin of the spin- $1 / 2$ photoluminescencedetected magnetic resonance in a $\pi$-conjugated polymer," Physical Review B, vol. 71, no. 24, pp. 1-11, 2005.

[83] M. K. Lee, M. Segal, Z. G. Soos, J. Shinar, and M. A. Baldo, "Yield of singlet excitons in organic light-emitting devices: a double modulation photoluminescence-detected magnetic resonance study," Physical Review Letters, vol. 94, no. 13, Article ID 137403 , 2005.

[84] S. Sinha and A. P. Monkman, "Delayed recombination of detrapped space-charge carriers in poly[2-methoxy-5- (2l ethyl-hexyloxy)-1,4-phenylene vinylene]-based light-emitting diode," Journal of Applied Physics, vol. 97, no. 11, Article ID 114505, pp. 1-7, 2005.

[85] S. Sinha, C. Rothe, R. Güntner, U. Scherf, and A. P. Monkman, "Electrophosphorescence and delayed electroluminescence from pristine polyfluorene thin-film devices at low temperature," Physical Review Letters, vol. 90, no. 12, Article ID 127402, 4 pages, 2003.

[86] J. S. Wilson, A. S. Dhoot, A. J. A. B. Seeley, M. S. Khan, A. Köhler, and R. H. Friend, "Spin-dependent exciton formation in $\pi$-conjugated compounds," Nature, vol. 413, no. 6858, pp. 828831, 2001.

[87] C. Rothe, S. King, and A. Monkman, "Long-range resonantly enhanced triplet formation in luminescent polymers doped with iridium complexes," Nature Materials, vol. 5, no. 6, pp. 463466, 2006.

[88] P. A. Lane, L. C. Palilis, D. F. O’Brien et al., "Origin of electrophosphorescence from a doped polymer light emitting diode," Physical Review B, vol. 63, no. 23, Article ID 235206, 8 pages, 2001.

[89] H. A. Al Attar and A. P. Monkman, "Dopant effect on the charge injection, transport, and device efficiency of an electrophosphorescent polymeric light-emitting device," Advanced Functional Materials, vol. 16, no. 17, pp. 2231-2242, 2006.

[90] L. C. Lin, H. F. Meng, J. T. Shy et al., "Triplet-to-singlet exciton formation in poly(p-phenylene-vinylene) light-emitting diodes," Physical Review Letters, vol. 90, no. 3, Article ID 036601, 4 pages, 2003.
[91] A. S. Dhoot, D. S. Ginger, D. Beljonne, Z. Shuai, and N. C. Greenham, "Triplet formation and decay in conjugated polymer devices," Chemical Physics Letters, vol. 360, no. 3-4, pp. 195-201, 2002.

[92] Y. Cao, I. D. Parker, G. Yu, C. Zhang, and A. J. Heeger, "Improved quantum efficiency for electroluminescence in semiconducting polymers," Nature, vol. 397, no. 6718, pp. 414-415, 1999.

[93] M. Tammer, R. W. T. Higgins, and A. P. Monkman, "High optical anisotropy in thin films of polyfluorene and its affect on the outcoupling of light in typical polymer light emitting diode structures," Journal of Applied Physics, vol. 91, no. 7, Article ID 4010, p. 4, 2002.

[94] E. J. W. List, R. Guentner, P. S. de Freitas, and U. Scherf, "The effect of keto defect sites on the emission properties of polyfluorene-type materials," Advanced Materials, vol. 14, pp. 374-378, 2002

[95] S. I. Hintschich, C. Rothe, S. Sinha, A. P. Monkman, P. Scandiucci de Freitas, and U. Scherf, "Population and decay of keto states in conjugated polymers," Journal of Chemical Physics, vol. 119, no. 22, pp. 12017-12022, 2003.

[96] H. Spreitzer, H. Becker, E. Breuning et al., "Light emitting polymer materials for full-color displays," in Organic LightEmitting Materials and Devices VI, pp. 16-25, usa, July 2002.

[97] A. Van Dijken, A. Perro, E. A. Meulenkamp, and K. Brunner, "The influence of a PEDOT:PSS layer on the efficiency of a polymer light-emitting diode," Organic Electronics, vol. 4, no. 2-3, pp. 131-141, 2003.

[98] S. M. King, D. Dai, C. Rothe, and A. P. Monkman, "Exciton annihilation in a polyfluorene: low threshold for singlet-singlet annihilation and the absence of singlet-triplet annihilation," Physical Review B, vol. 76, no. 8, Article ID 085204, 2007.

[99] M. Deussen, M. Scheidler, and H. Bässler, "Electric fieldinduced photoluminescence quenching in thin-film lightemitting diodes based on poly(phenyl-p-phenylene vinylene)," Synthetic Metals, vol. 73, no. 2, pp. 123-129, 1995.

[100] E. J. W. List, C. H. Kim, A. K. Naik et al., "Interaction of singlet excitons with polarons in wide band-gap organic semiconductors: a quantitative study," Physical Review B, vol. 64, no. 15, Article ID 155204, pp. 1552041-15520411, 2001.

[101] C. Rothe, H. A. Al Attar, and A. P. Monkman, "Absolute measurements of the triplet-triplet annihilation rate and the chargecarrier recombination layer thickness in working polymer lightemitting diodes based on polyspirobifluorene," Physical Review $B$, vol. 72, no. 15, pp. 1-9, 2005.

[102] D. Hertel, H. Bässler, R. Guentner, and U. Schert, "Triplet-triplet annihilation in a poly(fluorene)-derivative," Journal of Chemical Physics, vol. 115, no. 21, pp. 10007-10013, 2001.

[103] C. Rothe and A. P. Monkman, "Triplet exciton migration in a conjugated polyfluorene," Physical Review B, vol. 68, no. 7, Article ID 075208, pp. 752081-7520811, 2003.

[104] C. Rothe and A. Monkman, "Dynamics and trap-depth distribution of triplet excited states in thin films of the light-emitting polymer poly(9,9-di(ethylhexyl)fluorene)," Physical Review B, vol. 65, no. 7, Article ID 073201, pp. 0732011-0732014, 2002.

[105] P. W. M. Blom, M. J. M. De Jong, and J. J. M. Vleggaar, "Electron and hole transport in poly(p-phenylene vinylene) devices," Applied Physics Letters, vol. 68, no. 23, pp. 3308-3310, 1996.

[106] C. Rothe, S. M. King, and A. P. Monkman, "Electric-fieldinduced singlet and triplet exciton quenching in films of the 
conjugated polymer polyspirobifluorene," Physical Review B, vol. 72, no. 8, Article ID 085220, 2005.

[107] H. E. Lessing, A. Von Jena, and M. Reichert, "Triplet yield determination and heavy-atom effect from ground-state repopulation kinetics," Chemical Physics Letters, vol. 42, no. 2, pp. 218222, 1976.

[108] B. H. Wallikewitz, D. Kabra, S. Gelinas, and R. H. Friend, "Triplet dynamics in fluorescent polymer light-emitting diodes," Physical Review B, vol. 85, Article ID 045209, 15 pages, 2012.

[109] S. Sinha and A. P. Monkman, "Delayed electroluminescence via triplet-triplet annihilation in light emitting diodes based on poly[2-methoxy-5-(2I-ethyl-hexyloxy)-1,4-phenylene vinylene],' Applied Physics Letters, vol. 82, no. 26, pp. 4651-4653, 2003.

[110] C. Rothe and A. Monkman, "Regarding the origin of the delayed fluorescence of conjugated polymers," Journal of Chemical Physics, vol. 123, no. 24, Article ID 244904, pp. 1-6, 2005.

[111] S. M. King, M. Cass, M. Pintani et al., "The contribution of triplet-triplet annihilation to the lifetime and efficiency of fluorescent polymer organic light emitting diodes," Journal of Applied Physics, vol. 109, no. 7, Article ID 074502, 2011.

[112] C. A. Parker and C. G. Hatchard, "Delayed fluorescence of pyrene in ethanol," Transactions of the Faraday Society, vol. 59, pp. 284-295, 1963.

[113] J. Jortner, S. I. Choi, J. L. Katz, and S. A. Rice, “Triplet energy transfer and triplet-triplet interaction in aromatic crystals," Physical Review Letters, vol. 11, no. 7, pp. 323-326, 1963.

[114] J. B. Birks, "The quintet state of the pyrene excimer," Physics Letters A, vol. 24, no. 9, pp. 479-480, 1967.

[115] J. Saltiel, "Spin-statistical factors in reactions of free-radicals and triplet-states," Abstracts of Papers of the American Chemical Society, vol. 182, p. 65, 1981.

[116] J. Saltiel, G. R. Marchand, W. K. Smothers, S. A. Stout, and J. L. Charlton, "Concerning the spin-statistical factor in the triplet-triplet annihilation of anthracene triplets," Journal of the American Chemical Society, vol. 103, no. 24, pp. 7159-7164, 1981.

[117] M. B. Smith and J. Michl, "Singlet fission," Chemical Reviews, vol. 110, no. 11, pp. 6891-6936, 2010.

[118] R. Froese and K. Morokuma, "Accurate calculations of bondbreaking energies in $\mathrm{C}_{60}$ using the three-layered ONIOM method," Chemical Physics Letters, vol. 305305, no. 5-6, pp. 419424, 1999.

[119] W. G. Herkstroeter and P. B. Merkel, "The triplet state energies of rubrene and diphenylisobenzofuran," Journal of Photochemistry, vol. 16, no. 4, pp. 331-341, 1981.

[120] H. D. Burrows, J. Seixas de Melo, C. Serpa et al., “Triplet state dynamics on isolated conjugated polymer chains," Chemical Physics, vol. 285, no. 1, pp. 3-11, 2002.

[121] L. Ma, K. K. Zhang, C. Kloc, H. D. Sun, M. E. Michel-Beyerle, and G. G. Gurzadyan, "Singlet fission in rubrene single crystal: direct observation by femtosecond pump-probe spectroscopy," Physical Chemistry Chemical Physics, vol. 14, pp. 8307-8312, 2012.

[122] Y. Zhang, M. Whited, M. E. Thompson, and S. R. Forrest, "Singlet-triplet quenching in high intensity fluorescent organic light emitting diodes," Chemical Physics Letters, vol. 495, no. 4-6, pp. 161-165, 2010.

[123] R. W. T. Higgins, A. P. Monkman, H. G. Nothofer, and U. Scherf, "Energy transfer to porphyrin derivative dopants in polymer light-emitting diodes," Journal of Applied Physics, vol. 91, no. 1, pp. 99-105, 2002.
[124] Y. Iwasaki, T. Osasa, M. Asahi, M. Matsumura, Y. Sakaguchi, and T. Suzuki, "Fractions of singlet and triplet excitons generated in organic light-emitting devices based on a polyphenylenevinylene derivative," Physical Review B, vol. 74, no. 19, Article ID 195209, 2006.

[125] C. Rothe, K. Brunner, I. Bach, S. Heun, and A. P. Monkman, "Effects of triplet exciton confinement induced by reduced conjugation length in polyspirobifluorene copolymers," Journal of Chemical Physics, vol. 122, no. 8, Article ID 084706, pp. 1-6, 2005.

[126] F. Perrin, "La fluorescence des solutions," Annals of Physics, vol. 12, pp. 169-275, 1929.

[127] G. N. Lewis and M. Kasha, "Phosphorescence and the triplet state," Journal of the American Chemical Society, vol. 66, no. 12, pp. 2100-2116, 1944.

[128] J. C. Deaton, S. C. Switalski, D. Y. Kondakov et al., "E-type delayed fluorescence of a phosphine-supported cu 2( $\mu$-nar 2) 2 diamond core: harvesting singlet and triplet excitons in OLEDs," Journal of the American Chemical Society, vol. 132, no. 27, pp. 9499-9508, 2010.

[129] A. J. M. Miller, J. L. Dempsey, and J. C. Peters, "Long-lived and efficient emission from mononuclear amidophosphine complexes of copper," Inorganic Chemistry, vol. 46, no. 18, pp. 7244-7246, 2007.

[130] H. C. Longuet-Higgins and J. N. Murrell, "The electronic spectra of aromatic molecules $\mathrm{V}$ : the interaction of two conjugated systems," Proceedings of the Physical Society. Section A, vol. 68, no. 7, article no. 308, pp. 601-611, 1955.

[131] J. N. Murrell, "Relative importance of exciton delocalization and electron delocalization in polyene spectra," The Journal of Chemical Physics, vol. 37, no. 5, pp. 1162-1163, 1962.

[132] D. Chaudhuri, H. Wettach, K. J. Van Schooten et al., "Tuning the singlet-triplet gap in metal-free phosphorescent $\pi$-conjugated polymers," Angewandte Chemie, vol. 49, no. 42, pp. 7714-7717, 2010.

[133] A. Endo, K. Sato, K. Yoshimura et al., "Efficient up-conversion of triplet excitons into a singlet state and its application for organic light emitting diodes," Applied Physics Letters, vol. 98, no. 8, Article ID 083302, 2011.

[134] A. Endo, M. Ogasawara, A. Takahashi, D. Yokoyama, Y. Kato, and C. Adachi, "Thermally activated delayed fluorescence from Sn4+-porphyrin complexes and their application to organic light-emitting diodes -A novel mechanism for electroluminescence," Advanced Materials, vol. 21, no. 47, pp. 4802-4806, 2009.

[135] J. Kalinowski, "Excimers and exciplexes in organic electroluminescence," Materials Science- Poland, vol. 27, no. 3, pp. 735-756, 2009.

[136] S. A. Jenekhe and J. A. Osaheni, "Excimers and exciplexes of conjugated polymers," Science, vol. 265, no. 5173, pp. 765-768, 1994.

[137] K. Itano, H. Ogawa, and Y. Shirota, "Exciplex formation at the organic solid-state interface: yellow emission in organic light-emitting diodes using green-fluorescent tris(8-quinolinolato)aluminum and hole-transporting molecular materials with low ionization potentials," Applied Physics Letters, vol. 72, no. 6, pp. 636-638, 1998.

[138] M. Cocchi, D. Virgili, G. Giro et al., "Efficient exciplex emitting organic electroluminescent devices," Applied Physics Letters, vol. 80, no. 13, pp. 2401-2403, 2002. 
[139] J. Kalinowski, M. Cocchi, P. Di Marco, W. Stampor, G. Giro, and V. Fattori, "Impact of high electric fields on the charge recombination process in organic light-emitting diodes," Journal of Physics D, vol. 33, no. 19, pp. 2379-2387, 2000.

[140] L. C. Palilis, A. J. Mäkinen, M. Uchida, and Z. H. Kafafi, "Highly efficient molecular organic light-emitting diodes based on exciplex emission," Applied Physics Letters, vol. 82, no. 14, pp. 2209-2211, 2003.

[141] B. Frederichs and H. Staerk, "Energy splitting between triplet and singlet exciplex states determined with E-type delayed fluorescence," Chemical Physics Letters, vol. 460, no. 1-3, pp. 116$118,2008$.

[142] H. Beens and A. Weller, "Application of the tyablikov-bogolyubov diagonalization method to magnetic thin films," Acta Physica Polonica, vol. 34, pp. 539-541, 1968.

[143] A. Wellar, The Exciplex, Academic Press, New York, NY, USA, 1975.

[144] M. Cocchi, D. Virgili, C. Sabatini, and J. Kalinowski, "Organic electroluminescence from singlet and triplet exciplexes: exciplex electrophosphorescent diode," Chemical Physics Letters, vol. 421, no. 4-6, pp. 351-355, 2006.

[145] A. C. Morteani, A. S. Dhoot, J. S. Kim et al., "Barrier-Free Electron-Hole Capture in Polymer Blend Heterojunction LightEmitting Diodes," Advanced Materials, vol. 15, no. 20, pp. 17081712, 2003.

[146] K. Goushi, K. Yoshida, K. Sato, and C. Adachi, “Organic lightemitting diodes employing efficient reverse intersystem crossing for triplet-to-singlet state conversion," Nature Photonics, vol. 6, pp. 253-258, 2012.

[147] K. Goushi and C. Adachi, "Efficient organic light-emitting diodes through up-conversion from triplet to singlet excited states of exciplexes," Applied Physics Letters, vol. 101, Article ID 023306, 4 pages, 2012.

[148] V. Jankus, C. Chiang, F. B. Dias, and A. Monkman, "Deep blue exciplex organic light emitting diodes with enhanced efficiency through triplet fusion," Advanced Materials. In press.

[149] V. Jankus, C. Winscom, and A. P. Monkman, "Dynamics of triplet migration in films of $\mathrm{N}, \mathrm{N}^{\prime}$-diphenyl-N, $\mathrm{N}^{\prime}$-bis(1naphthyl)-1, 1'-biphenyl-4, $4^{\prime \prime}$-diamine," Journal of Physics Condensed Matter, vol. 22, no. 18, Article ID 185802, 2010.

[150] E. R. Bittner, I. Burghardt, and R. H. Friend, "Does interchain stacking morphology contribute to the singlet-triplet interconversion dynamics in polymer heterojunctions?" Chemical Physics, vol. 357, no. 1-3, pp. 159-162, 2009.

[151] A. C. Morteani, P. Sreearunothai, L. M. Herz, R. H. Friend, and C. Silva, "Exciton regeneration at polymeric semiconductor heterojunctions," Physical Review Letters, vol. 92, no. 24, Article ID 247402, 1 pages, 2004.

[152] A. C. Morteani, R. H. Friend, and C. Silva, "Endothermic exciplex-exciton energy-transfer in a blue-emitting polymeric heterojunction system," Chemical Physics Letters, vol. 391, no. 13, pp. 81-84, 2004.

[153] D. D. Gebler, Y. Z. Wang, J. W. Blatchford et al., "Exciplex emission in bilayer polymer light-emitting devices," Applied Physics Letters, vol. 70, no. 13, pp. 1644-1646, 1997. 

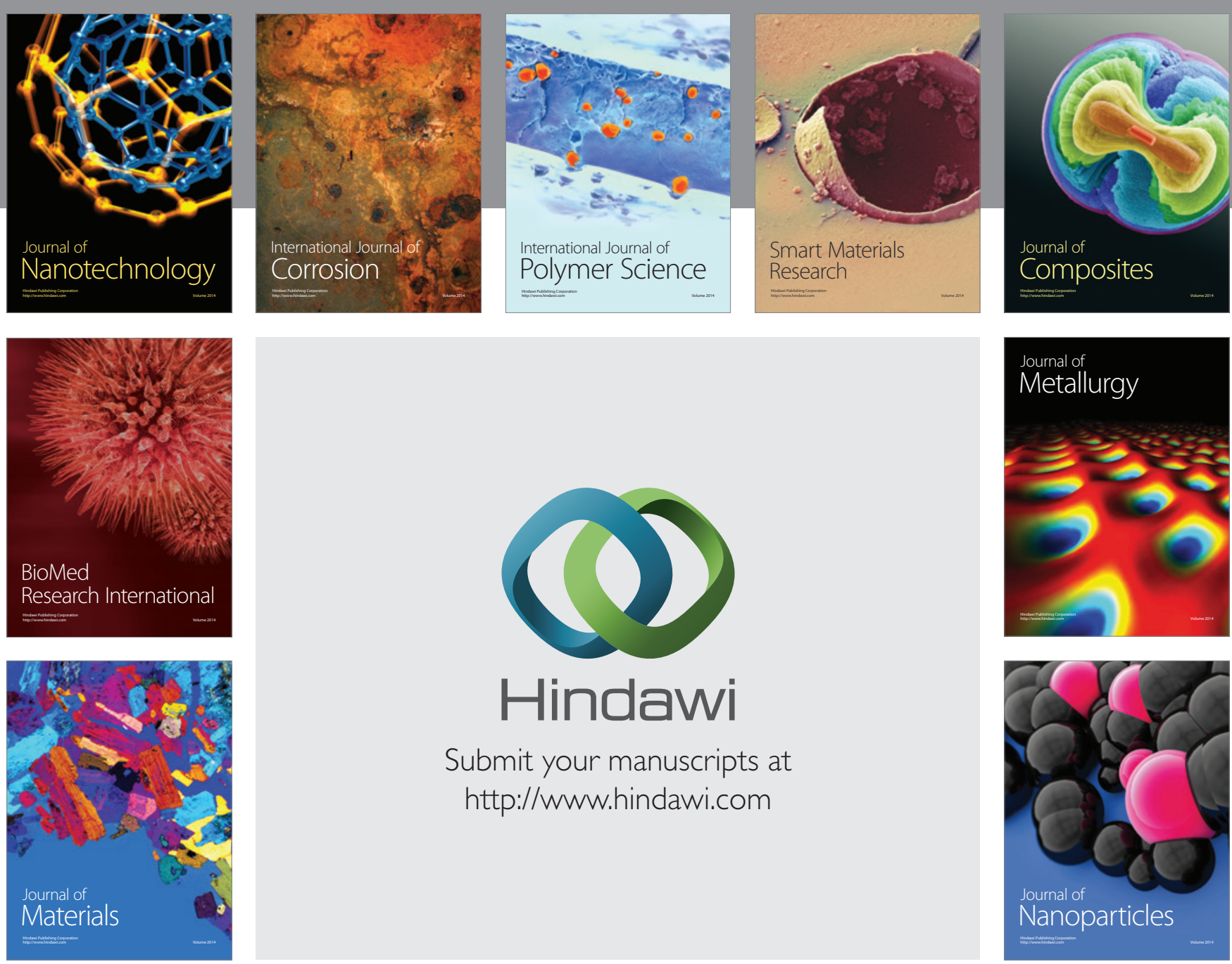

Submit your manuscripts at http://www.hindawi.com
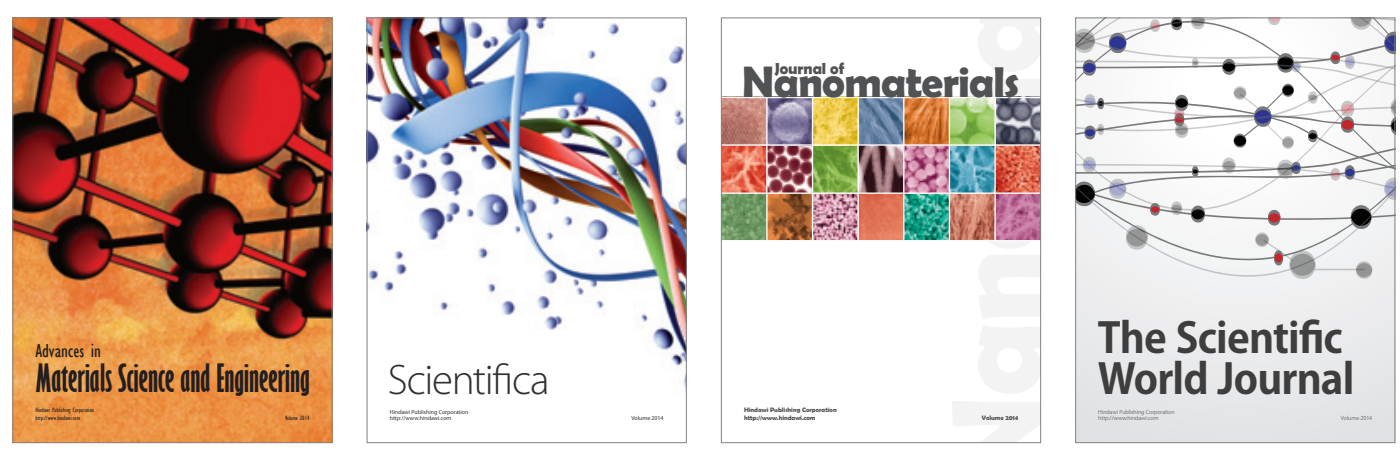

\section{The Scientific World Journal}
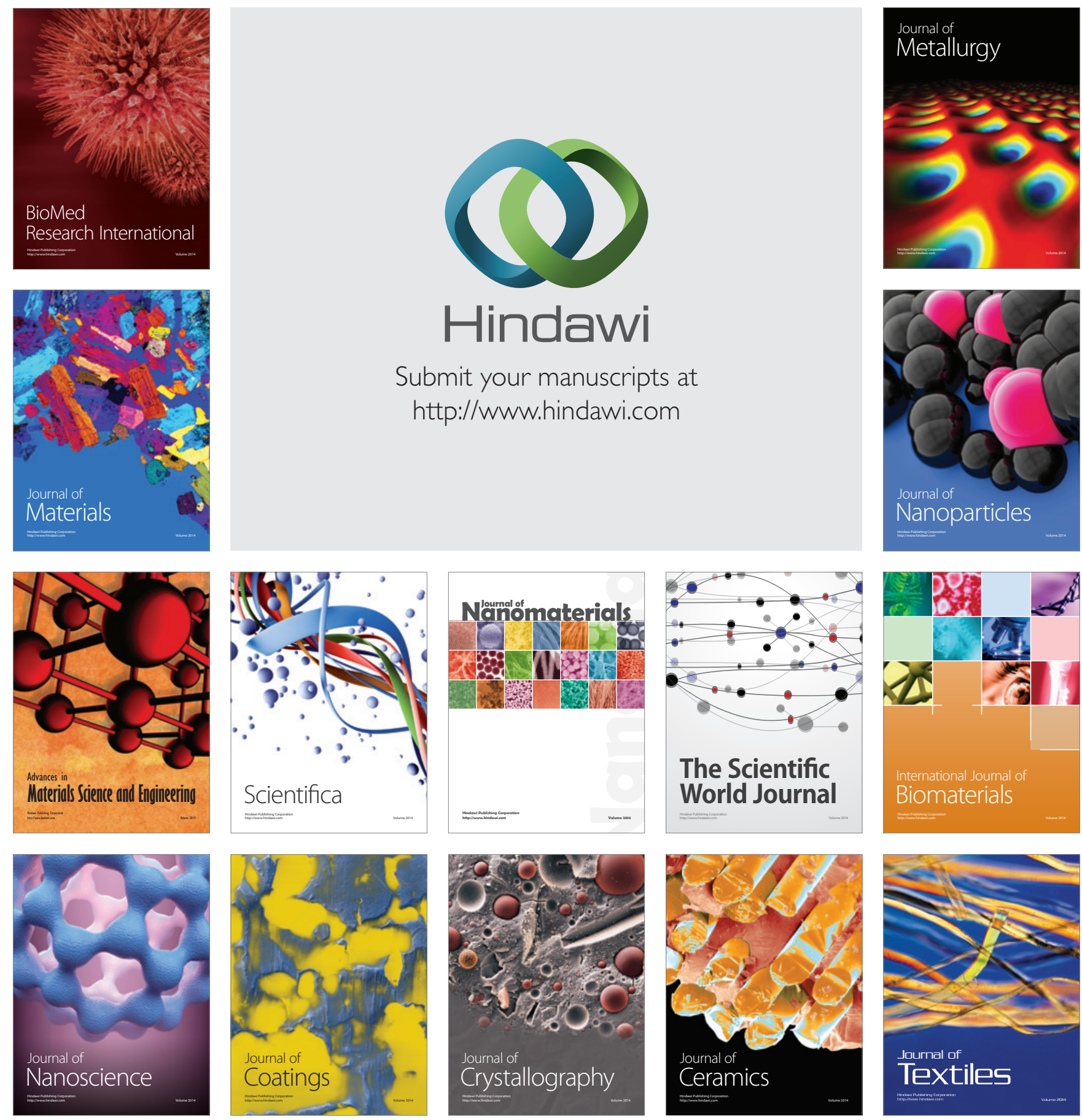